\title{
SUR LES CHIFFRES DES NOMBRES PREMIERS TRANSLATÉS
}

\author{
Mohamed Mkaouar, Najib Ouled Azaiez, Jörg M. Thuswaldner
}

\begin{abstract}
The aim of this work is to prove new results on a class of digital functions with special emphasis on shifted primes as arguments. Our method lies on the estimate of exponential sums of the form $\sum_{n \leqslant x} \Lambda(n) \exp \left(2 i \pi f\left(n+c_{n}\right)+\beta n\right)$ where $f$ a digital function, $\mathbf{c}=\left(c_{n}\right)$ is an almostperiodic sequence in $\mathbb{Z}$ and $\beta$ is a real parameter, which extend the works of Mauduit-Rivat [18] and Martin-Mauduit-Rivat [16] to the case of the shifted prime numbers satisfying a digital constraint.
\end{abstract}

Keywords: exponential sums, shifted primes, digital function.

\section{Introduction}

Dans tout ce travail, $\mathcal{P}$ désigne l'ensemble des nombres premiers. Notons $e(x)=$ $\exp (2 \pi i x),\|x\|$ la distance du nombre réel $x$ à l'entier le plus proche et pour $m_{1}, m_{2}$ entiers, $\left(m_{1}, m_{2}\right)$ est le plus grand commun diviseur de ces deux entiers. Si $f(n)=O(g(n))$, on écrit alors $f(n) \ll g(n)$, si de plus $g(n) \ll f(n)$, on écrit $f(n) \approx g(n)$. Nous désignons par $\Lambda$ la fonction de von Mangoldt définie pour tout nombre entier strictement positif $n$ par

$$
\Lambda(n)= \begin{cases}\log p & \text { si } n=p^{l} \text { avec } p \text { premier et } l \geqslant 1 \\ 0 & \text { sinon. }\end{cases}
$$

Notons $\pi(x)=\sharp\{p \leqslant x: p \in \mathcal{P}\}$ et $\pi(x, k, m)=\sharp\{p \leqslant x: p \in \mathcal{P}, p \equiv k(m)\}$. Soit $q$ un entier $\geqslant 2$, tout entier strictement positif $n$ admet une $q$-représentation unique sous la forme

$$
n=\sum_{j=0}^{\nu} n_{j} q^{j} \quad n_{j} \in\{0,1, \cdots, q-1\} \text { et } n_{\nu} \neq 0 .
$$

Conformément à l'usage, on désigne par $v_{q}(n)=v(n)$ la valuation $q$-adique de $n$ (i.e. $v(n)=\min \left\{j \leqslant \nu, n_{j} \neq 0\right\}$ ) et $d_{q}(n)=d(n)=\nu$ est le degré $q$-adique

2010 Mathematics Subject Classification: primary: 11A63, 11L03, 11N05; secondary: $11 \mathrm{~L} 20,11 \mathrm{~N} 60$ 
de $n$. Pour tout $0 \leqslant j \leqslant q-1$, on désigne par $|\cdot|_{j}$ la fonction comptant le nombre d'occurences du chiffre $j$ dans le développement en base $q$, soit

$$
|n|_{j}=\sharp\left\{0 \leqslant i \leqslant \nu \mid n_{i}=j\right\} .
$$

En particulier la fonction somme des chiffres en base $q$ est définie pour tout nombre entier positif $n$ par

$$
s(n)=s_{q}(n)=\sum_{j=0}^{\nu} n_{j}=\sum_{0 \leqslant j<q} j|n|_{j} .
$$

\subsection{Fonctions digitales}

La notion de fonction digitale a été étudiée par Drmota et Mauduit [6]. Il s'agit des fonctions $f: \mathbb{N} \rightarrow \mathbb{R}$ définies pour tout nombre entier positif $n$ par

$$
f(n)=\sum_{0 \leqslant j<q} a_{j}|n|_{j}
$$

où $a_{0}, a_{1}, \cdots, a_{q-1}$ sont des nombres réels. Il est à noter qu'une fonction digitale $f$ est complètement déterminée par ses valeurs $f(j), 0 \leqslant j<q$. De plus si $f(0)=0$, alors une telle fonction est appelée fonction fortement $q$-additive et vérifiant $f\left(a q^{j}\right)=f(a)$ pour tout $(a, j) \in \mathbb{N}^{2}$ (notion introduite par Bellman et Shapiro [2] et Gelfond [13]).

Notation 1.1. On note $\mathcal{F}$ l'ensemble des fonctions digitales, $\mathcal{F}^{\sharp}$ l'ensemble des fonctions digitales, $f(n)=\sum_{0 \leqslant j<q} a_{j}|n|_{j}$, tels que la suite $\alpha_{0}, \alpha_{1}, \cdots, a_{q-1}$ n'est pas une progression arithmétique modulo 1 dont la raison est un multiple entier de $1 /(q-1)$. On notera $\mathcal{F}_{0}$ l'ensemble des fonctions digitales $f(n)=\sum_{0 \leqslant j<q} a_{j}|n|_{j}$, dont la suite $a_{0}, a_{1}, \cdots, a_{q-1}$ est une progression arithmétique modulo 1 . Il est à noter que $\mathcal{F}=\mathcal{F}_{0} \cup \mathcal{F}^{\sharp}$.

Remarque 1.2. La fonction somme des chiffres $s()=.s_{q}()=.\sum_{1 \leqslant j<q} j|\cdot|_{j} \in \mathcal{F}_{0}$. De plus si $\alpha \in \mathbb{R} \backslash \mathbb{Q}$, alors $\alpha s_{q} \in \mathcal{F}_{0} \bigcap \mathcal{F}^{\sharp}$.

\subsection{Suite presque périodique}

Nous introduisons la notion de suite presque périodique qui est en quelque sorte une suite "bien approchée" par une suite périodique.

Définition 1.3. Soit $T \in \mathbb{N}^{*}$ et $0 \leqslant \theta<1$, une suite bornée $\left(c_{n}\right)$ est dite $(T, \theta)$-presque périodique s'il existe une suite $\left(e_{n}\right)$ purement $T$-périodique (suite $T$-périodiqe à partir du rang 0) telle que

$$
\sharp\left\{n \leqslant N: c_{n} \neq e_{n}\right\} \ll N^{\theta} .
$$

Dans ce cas, on dit que la suite $\left(c_{n}\right)$ est presque périodique. 
Exemple 1.4. Soient $\left(e_{n}\right)$ la suite nulle et $\left(c_{n}\right)$ la suite définie par

$$
c_{n}= \begin{cases}1 & \text { si } n \text { est un carré } \\ 0 & \text { sinon. }\end{cases}
$$

Il est clair que la suite $\left(c_{n}\right)$ n'est pas périodique, de plus

$$
\sharp\left\{n \leqslant N: c_{n} \neq e_{n}\right\}=N^{\frac{1}{2}}+O(1),
$$

ce qui donne que $c_{n}$ est $\left(1, \frac{1}{2}\right)$-presque périodique.

Remarque 1.5. Toute suite $T$-périodique est $(T, 0)$-presque périodique.

\subsection{Propriétés statistiques des suites arithmétiques}

La fonction somme des chiffres en base $q$ que nous désignons par $s=s_{q}$ définie par $s(n)=\sum_{j=0}^{\nu} n_{j}$, apparaît dans de nombreux problèmes mathématiques (voir [17] pour un survol de ses questions) mais c'est Mahler [15] qui la fait intervenir le premier dans un contexte d'analyse harmonique. Des résultats importants concernant la répartition dans les progressions arithmétiques de la somme des chiffres de certaines suites classiques ont été donnés par Gelfond dans [13] (on trouvera dans [10] des premiers résultats concernant le cas où $b$ est un nombre premier):

Théorème A. Soient $q, b$ et $d$ des nombres entiers positifs tels que $q \geqslant 2$ et $(b, q-1)=1$. Alors pour tout $(a, r) \in\{0, \cdots, b-1\} \times\{0, \cdots, d-1\}$, on a

$$
\operatorname{card}\left\{n<N, s_{q}(n) \equiv a(b), n \equiv r(d)\right\}=\frac{N}{b d}+O\left(N^{\lambda}\right)
$$

avec $\lambda=\frac{1}{2 \log q} \log \frac{q \sin \pi / 2 b}{\sin \pi / 2 b q}<1$.

Gelfond déduit de ce théorème la bonne répartition dans les progressions arithmétiques de la somme des chiffres des entiers sans facteurs puissance $k$-ième $(k \geqslant 2)$ et Mauduit et Sárközy en déduisent [20] que les nombres $s_{q}\left(p_{1}+p_{2}\right)_{p_{\text {b }} p_{2}<N, p_{i} \in \mathcal{P}}$ sont bien répartis dans les progressions arithmétiques (on trouvera également dans [20] des résultats concernant les valeurs moyennes et les valeurs extrémales du nombre de facteurs premier (comptés avec ou sans multiplicité) de l'entier $n$ lorsque celui-ci est soumis à la condition $s_{q}(n) \equiv a(b)$, ainsi qu'un théorème du type Erdös-Kac).

Par contre le théorème A n'est pas suffisant pour déduire la répartition de la somme des chiffres des nombres premiers, ce qui conduit Gelfond à poser plusieurs problèmes à la fin de son article, dont le suivant: "Il serait aussi intéressant de trouver le nombre des premiers $p \leqslant x$ tels que $s_{q}(p) \equiv l(b)$ " (voir [3] ,[11] et [12]) pour une réponse partielle dans le cas des nombres presque premiers.

En 2010, Mauduit et Rivat dans [18], donnent une réponse complète à la question de Gelfond [13] ainsi qu'à des questions de męme nature concernant les propriétés arithmétiques de la somme des chiffres des nombres premiers: 
Théorème B. Pour $q$ et $b$ entiers $\geqslant 2$, il existe $\sigma_{q, b}>0$ tel que pour tout $a \in \mathbb{Z}$, $\operatorname{card}\left\{p \leqslant x\right.$ tels que $\left.s_{q}(p) \equiv a(b)\right\}=\frac{(b, q-1)}{b} \pi(x, a,(b, q-1))+O_{q, b}\left(x^{1-\sigma_{q, b}}\right)$.

La clef de leur démonstration du théorème $\mathrm{B}$ consiste à estimer une certaine somme d'exponentielles à savoir:

Théorème C. Pour $q \geqslant 2$ et $\alpha$ tels que $(q-1) \alpha \in \mathbb{R} \backslash \mathbb{Z}$, il existe $\sigma_{q}(\alpha)>0$ tel que

$$
\sum_{n \leqslant x} \Lambda(n) e\left(\alpha s_{q}(n)\right)=O_{q, \alpha}\left(x^{1-\sigma_{q}(\alpha)}\right)
$$

Il est à noter que l'estimation donnée dans le Théorème $\mathrm{C}$ a été améliorée dans [5] pour une estimation uniforme en $\alpha$. Ce qui leur permet par la suite de montrer dans [18]:

Théorème D. Pour $q \geqslant 2$, la suite $\left(\alpha s_{q}(p)\right)_{p \in \mathcal{P}}$ est équirépartie modulo 1 si et seulement si $\alpha \in \mathbb{R} \backslash \mathbb{Q}$.

Lorsque $f$ est une fontion digitale: $f=\sum_{0 \leqslant k<q} a_{k}|n|_{k} \in \mathcal{F}^{\sharp}$, on définit le réel

$$
\lambda_{q}(f)= \begin{cases}c_{1 q} \min _{t \in \mathbb{R}} \sum_{0 \leqslant j<i<q}\left\|a_{i}-a_{j}-(i-j) t\right\|^{2} & \text { si } f \notin \mathcal{F}_{0}, \\ c_{2 q}\left\|(q-1)\left(a_{1}-a_{0}\right)\right\|^{2} & \text { si } f \in \mathcal{F}_{0} \bigcap \mathcal{F}^{\sharp},\end{cases}
$$

où les $c_{i q}$ sont des constantes $>0$ ne dépendant que de $q$ (les $c_{i q}$ sont données explicitement en fonction de $q$ voir [16]). Il a été démontré récemment par Martin, Mauduit et Rivat [16] que $\lambda_{q}(f)>0$, il est à signaler aussi que si $f \in \mathcal{F}_{0} \backslash \mathcal{F}^{\sharp}$, alors $\lambda_{q}(f)=0$. De plus ils ont étendu les théorèmes de Hadamard-de la Vallée Poussin et de Vinogradov (voir [14], [7], [22]) au cas des nombres premiers vérifiant une contrainte digitale. Leur méthode repose en particulier sur l'estimation d'une somme d'exponentielles donnée par

Théorème E. Soient $q \geqslant 2, f \in \mathcal{F}^{\sharp}$. Alors pour tout $x \geqslant 2$ et $\beta \in \mathbb{R}$, on a

$$
\sum_{n \leqslant x} \Lambda(n) e(f(n)+\beta n) \ll\left(\log ^{4} x\right) x^{1-\lambda_{q}(f)},
$$

la constante implicite ne dépend que de $q, \lambda_{q}(f)$ définie dans (1.2).

\subsection{Description des résultats}

Les résultats que nous présentons s'inscrivent dans le cadre de l'étude de la $q$-représentation des nombres premiers translatés $\left(p+c_{p}, p:\right.$ premier, $\mathbf{c}=\left(c_{n}\right)$ : est une suite presque périodique à valeur dans $\mathbb{Z})$. L'objectif de la section 2 est l'étude d'une somme d'exponentielles de type

$$
\sum_{n \leqslant x} \Lambda(n) e\left(f\left(n+c_{n}\right)+\beta n\right),
$$


où $f()=.\sum a_{k}|\cdot|_{k}$ et $\mathbf{c}=\left(c_{n}\right)$ est une suite presque périodique. Il est à noter qu'une estimation est faite sur une somme de type (1.4) avec $\mathbf{c}=\left(c_{n}\right)$ qui est une suite "régulière". L'estimation de la somme (1.4), nous permet d'étudier l'équirépartition de la suite $\left(\alpha f\left(p+c_{p}\right)\right)_{p \in \mathcal{P}}$. A la fin de cette section, nous généralisons le problème ternaire de Goldbach (voir par exemple [4]) amélioré récemment par Martin-Mauduit-Rivat [16].

Dans la section 3, une étude de la structure arithmétique et statistique de l'ensemble $\left\{p+c_{p} \leqslant x: p \in \mathcal{P}, f\left(p+c_{p}\right) \equiv a(b)\right\}$ nous permet de donner un théorème de type Gelfond qui assure la bonne répartition dans les progressions arithmétiques de $\sum a_{k}|n|_{k}$, (où la suite $\mathbf{a}=\left(a_{k}\right)$ est à valeurs dans $\mathbb{Z}$ ).

\section{Somme d'exponentielles et formules asymptotiques}

Pour estimer la somme d'exponentielles donnée par (1.4), nous introduisons la suite $\gamma(\mathbf{a}, n, i)=a_{n_{i}+1}-a_{n_{i}}$ et nous commençons dans cette section par l'étude de cette suite.

\subsection{Etude de la suite $\gamma(\mathbf{a}, \boldsymbol{n}, \boldsymbol{u})$}

Soient $\mathbf{a}=\left(a_{k}\right)_{0 \leqslant k<q}$ une suite de nombres réels (on convient que $a_{q}=a_{0}$ ), $n=\sum_{i=0}^{\nu} n_{i} q^{i}$ (on convient que $n_{j}=0$ pour $j>\nu$ et par suite, on se permet d'écrire $\left.n=\sum_{i=0}^{+\infty} n_{i} q^{i}\right)$ et

$$
\gamma(\mathbf{a}, n, i)=a_{n_{i}+1}-a_{n_{i}}
$$

Lemme 2.1. Soit $u \in \mathbb{N}$, alors la suite $\left(n_{u}\right)_{n \in \mathbb{N}}$ est $q^{u+1}-$ périodique. De plus si $u \in \mathbb{N}^{*}, 0 \leqslant l<q$ et $n \in \mathbb{N}$, alors on $a(q n+l)_{u}=(n)_{u-1}$.

Démonstration. Soient $n=\sum_{i=0}^{+\infty} n_{i} q^{i}$ et $n+q^{u+1}=\sum_{i=0}^{+\infty}\left(n+q^{u+1}\right){ }_{i} q^{i}$, les développements $q$-adique respectivement de $n$ et $n+q^{u+1}$. Alors

$$
n+q^{u+1}=\sum_{i=0}^{u} n_{i} q^{i}+q^{u+1} N,
$$

où $N=1+\sum_{i=u+1}^{+\infty} n_{i} q^{i-u-1}$, ce qui donne d'après l'unicité de l'écriture $q$-adique de $n+q^{u+1}$,

$$
\left(n+q^{u+1}\right)_{u}=n_{u} .
$$

D'autre part, $l+q n=l+\sum_{i=0}^{+\infty} n_{i} q^{i+1}=l+\sum_{i=1}^{+\infty} n_{i-1} q^{i}$, ce qui donne par conséquent $(q n+l)_{u}=(n)_{u-1}$.

Il est clair d'après le lemme 2.1 que si $u \in \mathbb{N}$ (fixé), $\varepsilon \in\{ \pm 1\}$, alors la fonction $n \mapsto \varepsilon \gamma(\mathbf{a}, n, u)$ est périodique de période $q^{u+1}$. De plus si $u \in \mathbb{N}^{*}, 0 \leqslant l<q$ et $n \in \mathbb{N}$, alors on a $\gamma(\mathbf{a}, q n+l, u)=\gamma(\mathbf{a}, n, u-1)$. 
On considère la transformée de Fourier discrète de la suite $(\varepsilon \gamma(\mathbf{a}, n, u))_{n \geqslant 0}$,

$$
F_{\mathbf{a}, u, \varepsilon}(h)=\frac{1}{q^{u+1}} \sum_{0 \leqslant k<q^{u+1}} e\left(\varepsilon \gamma(\mathbf{a}, k, u)-\frac{k h}{q^{u+1}}\right), \quad h \in \mathbb{Z}
$$

et

$$
F_{\mathbf{a}, 0, \varepsilon}(h)=\frac{1}{q} \sum_{0 \leqslant k<q} e\left(\varepsilon\left(a_{k+1}-a_{k}\right)-\frac{k h}{q}\right), \quad h \in \mathbb{Z} .
$$

En remplaçant dans (2.2) l'entier $k$ par $q k+l$ avec $0 \leqslant k<q^{u}$ et $0 \leqslant l<q$, on obtient

$$
\begin{aligned}
F_{\mathbf{a}, u, \varepsilon}(h) & =\frac{1}{q^{u+1}} \sum_{0 \leqslant l<q} e\left(-\frac{l h}{q^{u+1}}\right) \sum_{0 \leqslant k<q^{u}} e\left(\varepsilon \gamma(\mathbf{a}, k, u-1)-\frac{k h}{q^{u}}\right) \\
& =\frac{1}{q}\left\{\sum_{0 \leqslant l<q} e\left(-\frac{l h}{q^{u+1}}\right)\right\} F_{\mathbf{a}, u-1, \varepsilon}(h) \\
& =\frac{1}{q^{u}} \prod_{j=1}^{u}\left(\sum_{0 \leqslant l<q} e\left(-\frac{l h}{q^{j+1}}\right)\right) F_{\mathbf{a}, 0, \varepsilon}(h),
\end{aligned}
$$

or pour $j \in \mathbb{N}^{*}$ et $h \in \mathbb{Z}$,

$$
\left|\sum_{0 \leqslant l<q} e\left(-\frac{l h}{q^{j+1}}\right)\right|= \begin{cases}\left|\frac{\sin \frac{h \pi}{q^{j}}}{\sin \frac{h \pi}{q^{j+1}}}\right| & \text { si } q^{j+1} \nmid h \\ q & \text { sinon. }\end{cases}
$$

Il est clair que si $j \geqslant 1$ et $q^{j} \| h$, alors

$$
F_{\mathbf{a}, j, \varepsilon}(h)=\left(\sum_{0 \leqslant l<q} e\left(-\frac{l h}{q^{j+1}}\right)\right) F_{\mathbf{a}, j-1, \varepsilon}(h)=0 .
$$

Ce qui donne avec (2.4) et (2.5)

$$
\left|F_{\mathbf{a}, u, \varepsilon}(h)\right|= \begin{cases}\frac{1}{q^{u}}\left|F_{\mathbf{a}, 0, \varepsilon}(h)\right| \mid \prod_{j=1}^{u} \frac{\sin \frac{h \pi}{q^{j}}}{\sin \frac{h \pi}{q^{j+1}} \mid} & \text { si } q \nmid h \\ 0 & \text { sinon. }\end{cases}
$$

Ce qui donne en particulier, pour tout $0<h<q^{u+1}$,

$$
\left|F_{\mathbf{a}, u, \varepsilon}(h)\right| \leqslant \frac{1}{q^{u}}\left|\frac{\sin \frac{h \pi}{q}}{\sin \frac{h \pi}{q^{u+1}}}\right|\left|F_{\mathbf{a}, 0, \varepsilon}(h)\right| \quad \text { et } F_{\mathbf{a}, u, \varepsilon}(0)=F_{\mathbf{a}, 0, \varepsilon}(0) .
$$

Les calculs ultérieurs nécessitent un type de renseignement spécifique concernant la fonction $F_{\mathbf{a}, u, \varepsilon}$ à savoir une majoration en moyenne de $F_{\mathbf{a}, u, \varepsilon}$. 
Lemme 2.2. Soit $m \in \mathbb{N}^{*}$, alors

$$
\sum_{1 \leqslant k<m} \frac{1}{\sin \frac{k \pi}{m}} \leqslant m \log m
$$

Démonstration. Pour une fonction convexe $f$,

$$
f(s) \leqslant \frac{1}{\alpha} \int_{s-\frac{1}{2} \alpha}^{s+\frac{1}{2} \alpha} f(t) d t
$$

donc pour $f(s)=\frac{1}{\sin \pi x}$ et $\alpha=\frac{1}{m}$, nous obtenons

$$
\begin{aligned}
\sum_{1 \leqslant k<m} \frac{1}{\sin \frac{k \pi}{m}} & =m \sum_{1 \leqslant k<m} \int_{\frac{k}{m}-\frac{1}{2 m}}^{\frac{k}{m}+\frac{1}{2 m}} \frac{d t}{\sin \pi t} \\
& =m \int_{\frac{1}{2 m}}^{1-\frac{1}{2 m}} \frac{d t}{\sin \pi t}=2 m \int_{\frac{1}{2 m}}^{\frac{1}{2}} \frac{d t}{\sin \pi t} \\
& \leqslant 2 m \int_{\frac{1}{2 m}}^{\frac{1}{2}} \frac{d t}{2 t}=m \log m,
\end{aligned}
$$

la dernière inégalité vient du fait que $\sin \pi t>2 t$, pour $0<t<\frac{1}{2}$.

Lemme 2.3. Pour $u \in \mathbb{N}, \varepsilon \in\{ \pm 1\}$, on $a$

$$
\sum_{0 \leqslant h<q^{u+1}}\left|F_{\mathbf{a}, u, \varepsilon}(h)\right| \leqslant(u+2) q \log q .
$$

Démonstration. Compte-tenu de l'expression de $F_{\mathbf{a}, 0, \varepsilon}(h)$ dans $(2.3)$, on obtient $\left|F_{\mathbf{a}, 0, \varepsilon}(h)\right| \leqslant 1$, et d'après l'expression de $F_{\mathbf{a}, u, \varepsilon}(h)$ dans $(2.6)$, il vient que

$$
\begin{aligned}
\sum_{0 \leqslant h<q^{u+1}}\left|F_{\mathbf{a}, u, \varepsilon}(h)\right| & \leqslant 1+\frac{1}{q^{u}} \sum_{1 \leqslant h<q^{u+1}}\left|\frac{\sin \frac{h \pi}{q}}{\sin \frac{h \pi}{q^{u+1}}}\right| \\
& \leqslant 1+\frac{1}{q^{u}} \sum_{1 \leqslant h<q^{u+1}} \frac{1}{\sin \frac{h \pi}{q^{u+1}}}
\end{aligned}
$$

ce qui donne d'après le lemme 2.2

$$
\sum_{0 \leqslant h<q^{u+1}}\left|F_{\mathbf{a}, u, \varepsilon}(h)\right| \leqslant 1+q \log \left(q^{u+1}\right) \leqslant(u+2) q \log q .
$$

\subsection{Estimation d'une somme d'exponentielles}

L'objectif de cette partie est d'exploiter la majoration en moyenne de $F_{\mathbf{a}, u, \varepsilon}$ pour donner une estimation de la somme

$$
H_{q}(x, f, \beta, \mathbf{c})=\sum_{\|\mathbf{c}\|<n \leqslant x} \Lambda(n) e\left(f\left(n+c_{n}\right)+\beta n\right),
$$

où $\mathbf{c}=\left(c_{n}\right)_{n \geqslant 0}$ est une suite presque périodique à valeur dans $\mathbb{Z}$ et $\|\mathbf{c}\|=\max \left|c_{i}\right|$. 
Lemme 2.4. Soient $\mathbf{a}=\left(a_{k}\right)_{0 \leqslant k<q}$ une suite de nombres réels et $f=\sum_{k=0}^{q-1} a_{k}|\cdot|_{k}$ $\in \mathcal{F}$. Alors pour tout $n=\sum_{i=0}^{\nu} n_{i} q^{i} \in \mathbb{N}^{*}$ (on convient que $n_{j}=0$ dès que $j>\nu$ ),

$$
\begin{aligned}
f(n+1)= & f(n)+a_{n_{v(n+1)}+1}-a_{n_{v(n+1)}}+\left(a_{0}-a_{q-1}\right) v(n+1) \\
& +a_{0}(d(n+1)-d(n)) \\
= & f(n)+\gamma(\mathbf{a}, n, v(n+1))+\left(a_{0}-a_{q-1}\right) v(n+1) \\
& +a_{0}(d(n+1)-d(n)) .
\end{aligned}
$$

où $v(n)$ est la valuation $q$-adique de $n$ et $d(n)$ est son degré q-adique.

Démonstration. Soit $n=\sum_{i=0}^{\nu} n_{i} q^{i}, n_{\nu} \neq 0$.

- Si $v(n+1) \leqslant \nu=d(n)$, il est clair que $n_{v(n+1)} \neq q-1$ et que

$$
n=n_{\nu} q^{\nu}+\cdots+n_{v(n+1)} q^{v(n+1)}+(q-1)\left(q^{v(n+1)-1}+\cdots+1\right),
$$

et

$$
n+1=n_{\nu} q^{\nu}+\cdots+\left(n_{v(n+1)}+1\right) q^{v(n+1)},
$$

ce qui nous permet d'écrire

$$
f(n+1)=f(n)+a_{n_{v(n+1)}+1}-a_{n_{v(n+1)}}+\left(a_{0}-a_{q-1}\right) v(n+1)
$$

et comme dans ce cas $d(n+1)=d(n)$, alors

$$
\begin{aligned}
f(n+1)= & f(n)+a_{n_{v(n+1)}+1}-a_{n_{v(n+1)}}+\left(a_{0}-a_{q-1}\right) v(n+1) \\
& +a_{0}(d(n+1)-d(n)) .
\end{aligned}
$$

- Si $v(n+1)=\nu+1=d(n)+1$, alors

$$
n=(q-1)\left(q^{\nu}+q^{\nu-1}+\cdots+q+1\right) \text { et } n+1=q^{\nu+1},
$$

ce qui donne

$$
f(n)=v(n+1) a_{q-1} \text { et } f(n+1)=a_{1}+a_{0} v(n+1),
$$

ce qui donne

$$
f(n+1)=f(n)+a_{1}+\left(a_{0}-a_{q-1}\right) v(n+1),
$$

comme $n_{v(n+1)}=0$ et $d(n+1)=\nu+1=d(n)+1$, alors

$$
\begin{aligned}
f(n+1)= & f(n)+a_{n_{v(n+1)}+1}-a_{n_{v(n+1)}}+\left(a_{0}-a_{q-1}\right) v(n+1) \\
& +a_{0}(d(n+1)-d(n)) .
\end{aligned}
$$

Ce qui donne dans tous les cas la relation (2.11) est toujours vérifiée. 
Remarque 2.5. Il est à signaler que pour $n \in \mathbb{N}^{*}$

$$
d(n+1)-d(n)= \begin{cases}1 & \text { si } n=q^{l}-1 \\ 0 & \text { sinon }\end{cases}
$$

Dans la suite on introduit pour chaque fonction digitale $f=\sum_{k=0}^{q-1} a_{k}|\cdot|_{k} \in \mathcal{F}$ une suite auxilière $(\tilde{f}(n))_{n \geqslant 1}$ définie par:

$$
\left\{\begin{array}{l}
\tilde{f}(1)=f(1) \\
\widetilde{f}(n+1)=\widetilde{f}(n)+\gamma(\mathbf{a}, n, v(n+1))+\left(a_{0}-a_{q-1}\right) v(n+1) .
\end{array}\right.
$$

Il est clair d'après la relation (2.12), que

$$
\tilde{f}(n-1)=\tilde{f}(n)-\gamma(\mathbf{a}, n-1, v(n))-\left(a_{0}-a_{q-1}\right) v(n) \quad \text { pour } n \geqslant 2 .
$$

Nous en déduisons pour $\varepsilon \in\{ \pm 1\}$ et $\mu=\frac{1+\varepsilon}{2}$ et $n+\varepsilon>0$.

$$
\widetilde{f}(n+\varepsilon)=\widetilde{f}(n)+\varepsilon \gamma(\mathbf{a}, n+\varepsilon-\mu, v(n+\mu))+\varepsilon\left(a_{0}-a_{q-1}\right) v(n+\mu) .
$$

Plus généralement, si $c \in \mathbb{N}^{*}$, alors en appliquant $c$ fois la relation (2.13), on obtient pour $n+\varepsilon c>0$,

$$
\widetilde{f}(n+c \varepsilon)=\widetilde{f}(n)+\varepsilon \Gamma(\mathbf{a}, n, c, \varepsilon)+\varepsilon\left(a_{0}-a_{q-1}\right) V(n, c, \varepsilon)
$$

où

$$
\begin{aligned}
& \Gamma(\mathbf{a}, n, c, \varepsilon)=\Gamma(\mathbf{a}, n, c-1, \varepsilon)+\gamma(\mathbf{a}, n+\varepsilon c-\mu, v(n+\varepsilon(c-1)+\mu)) \\
& \Gamma(\mathbf{a}, n, 0, \varepsilon)=0
\end{aligned}
$$

et

$$
\begin{aligned}
& V(n, c, \varepsilon)=V(n, c-1, \varepsilon)+v(n+\varepsilon(c-1)+\mu) \\
& V(n, 0, \varepsilon)=0 .
\end{aligned}
$$

Remarque 2.6. Il est clair que si a $=\left(a_{k}\right)_{0 \leqslant k<q}, \widetilde{\mathbf{a}}=\left(\widetilde{a_{k}}\right)_{0 \leqslant k<q}=\left(a_{k}-a_{0}\right)_{0 \leqslant k<q}$ et $f=\sum_{k=0}^{q-1} a_{k}|\cdot|_{k} \in \mathcal{F}$, alors d'après les relations (2.11) et (2.12), on obtient

$$
f(n)=\widetilde{f}(n)+a_{0} d(n) .
$$

De plus $\tilde{f}=\sum_{k=0}^{q-1} \widetilde{a}_{k}|\cdot|_{k} \in \mathcal{F}$ et d'après les relations (1.2) et (2.1), on obtient

$$
\lambda_{q}(\widetilde{f})=\lambda_{q}(f) \quad \text { et } \gamma(\mathbf{a}, n, i)=\gamma(\widetilde{\mathbf{a}}, n, i) .
$$

Afin d'estimer maintenant la somme $H_{q}(x, f, \beta, \mathbf{c})$ dans $(2.10)$, nous allons utiliser le lemme suivant. 
Lemme 2.7. Soient $f \in \mathcal{F}, \alpha \in \mathbb{R}^{+}, \lambda>0$ et $\mathbf{c}$ une suite constante de terme général $c \in \mathbb{N}$. Alors

$$
H_{q}(x, \tilde{f}, \beta, \mathbf{c}) \ll\left(\log ^{\alpha} x\right) x^{1-\lambda} \Longleftrightarrow H_{q}(x, f, \beta, \mathbf{c}) \ll\left(\log ^{\alpha} x\right) x^{1-\lambda} .
$$

Démonstration. $\Longrightarrow)$ Soit $x>c$ et $N=\left\lfloor\log _{q} x\right\rfloor$, alors d'après la relation $(2.17)$

$$
\begin{aligned}
H_{q}(x, f, \beta, \mathbf{c}) & =\sum_{c<n \leqslant x} \Lambda(n) e(f(n+c)+\beta n) \\
& =\sum_{c<n \leqslant x} \Lambda(n) e\left(\tilde{f}(n+c)+a_{0} d(n+c)+\beta n\right)
\end{aligned}
$$

En découpant l'intervalle $] c, x]$ en tranche de type $\left[q^{k}, q^{k+1}[\right.$ de telle sorte que $d(n+c)=k$ si $n+c \in\left[q^{k}, q^{k+1}[\right.$, on obtient

$$
\begin{aligned}
H_{q}(x, f, \beta, \mathbf{c})= & \sum_{k=1}^{N-1} e\left(a_{0} k\right) \sum_{q^{k} \leqslant n+c<q^{k+1}} \Lambda(n) e(\widetilde{f}(n+c)+\beta n) \\
& +e\left(a_{0} N\right) \sum_{q^{N} \leqslant n+c \leqslant x} \Lambda(n) e(\tilde{f}(n+c)+\beta n)+O(1) \\
& \ll \sum_{k=1}^{N}\left|H_{q}\left(q^{k}-c, \tilde{f}, \beta, \mathbf{c}\right)\right|+\left|H_{q}(x-c, \tilde{f}, \beta, \mathbf{c})\right|+\left|H_{q}\left(q^{N}-c, \widetilde{f}, \beta, \mathbf{c}\right)\right| \\
& \ll \sum_{k=1}^{N}\left(\log ^{\alpha}\left(q^{k}-c\right)\right)\left(q^{k}-c\right)^{1-\lambda}+\left(\log ^{\alpha} x\right)(x-c)^{1-\lambda} \\
& \ll \sum_{k=1}^{N} k^{\alpha} q^{k(1-\lambda)}+\left(\log ^{\alpha} x\right) x^{1-\lambda} \\
& \ll\left(\log ^{\alpha} x\right) x^{1-\lambda} .
\end{aligned}
$$

$\Longleftarrow)$ La réciproque est analogue à ce qui précède quitte à remplacer $f$ par $\widetilde{f}$ et $a_{0}$ par $-a_{0}$ (ceci découle de la relation $(2.17)$ ).

Soient alors pour $c \in \mathbb{N}, \varepsilon \in\{ \pm 1\}, \alpha, \beta \in \mathbb{R}, f=\sum_{k=0}^{q-1} a_{k}|n|_{k} \in \mathcal{F}, \mathbf{b}=$ $\left(b_{n}\right)_{0 \leqslant n<q}$ une suite réelle (on convient que $b_{q}=b_{0}$ ) et pour $x \geqslant 2$,

$$
\begin{gathered}
g(n, \tilde{f}, \mathbf{b}, \alpha, \beta, c, \varepsilon)=\Lambda(n) e(\tilde{f}(n)+\beta n+\varepsilon \Gamma(\mathbf{b}, n, c, \varepsilon)-\varepsilon \alpha V(n, c, \varepsilon)) \\
G(x, \tilde{f}, \mathbf{b}, \alpha, \beta, c, \varepsilon)=\sum_{c<n \leqslant x} g(n, \tilde{f}, \mathbf{b}, \alpha, \beta, c, \varepsilon)
\end{gathered}
$$




\section{Remarque 2.8.}

(i) En utilisant les expressions de $\Gamma, V, g$ dans (2.15), (2.16) et (2.19), on obtient pour $c \in \mathbb{N}^{*}, \varepsilon \in\{ \pm 1\}$ et $\mu=\frac{1+\varepsilon}{2}$,

$$
\begin{aligned}
g(n, \tilde{f}, \mathbf{b}, \alpha, \beta, c, \varepsilon)= & g(n, \tilde{f}, \mathbf{b}, \alpha, \beta, c-1, \varepsilon) \\
& \times \psi(n+(c-1) \varepsilon+\mu, v(n+(c-1) \varepsilon+\mu)) .
\end{aligned}
$$

où $\psi(w, u)=e(\varepsilon \gamma(\mathbf{b}, w-1, u)-\varepsilon \alpha u)$.

(ii) Il est clair d'après $(2.14)$ que si $\mathbf{b}=\mathbf{a}$ et $\alpha=a_{q-1}^{\tilde{q}}$ et $\mathbf{c}=\left(c_{n}\right)$ la suite dont le terme général $c_{n}=c$, on a bien

$$
\begin{aligned}
G\left(x, \tilde{f}, \mathbf{a}, a_{q-1}, \beta, c, \varepsilon\right) & =\sum_{c<n \leqslant x} \Lambda(n) e(\tilde{f}(n+\varepsilon c)+\beta n) \\
& =H_{q}(x, \tilde{f}, \beta, \varepsilon \mathbf{c}) .
\end{aligned}
$$

Lemme 2.9. Soient $\alpha \in \mathbb{R}, q \geqslant 2, f \in \mathcal{F}^{\sharp}$ et $\mathbf{b}=\left(b_{n}\right)_{0 \leqslant n<q}$ une suite réelle. Alors pour tout $\beta \in \mathbb{R}, \varepsilon \in\{ \pm 1\}, c \in \mathbb{N}$ et $x \geqslant 2$,

$$
G(\beta, c)=G(x, \tilde{f}, \mathbf{b}, \alpha, \beta, c, \varepsilon) \ll\left(\log ^{2 c+4} x\right) x^{1-\lambda_{q}(f)} .
$$

La constante implicite ne dépend que de $c$ et $q, \lambda_{q}(f)$ est la constante définie dans (1.2).

Démonstration. La preuve se fait par récurrence sur $c$, le théorème $\mathrm{E}$ et la relation (2.18) fournissent le cas $c=0$.

Soit maintenant $c \geqslant 1, N=\left\lfloor\log _{q}(x+(c-1) \varepsilon+\mu)\right\rfloor, f()=.\sum_{0 \leqslant k<q} a_{k}|\cdot|_{k}$ et

$$
\psi(w, u)=e(\varepsilon \gamma(\mathbf{b}, w-1, u)-\varepsilon \alpha u),
$$

où $\gamma$ est la suite définie dans (2.1). En utilisant l'expression de $G$ dans (2.20) et celle de $g$ dans la remarque $2.8 i$ ), on obtient

$$
\begin{aligned}
G(\beta, c)= & \sum_{c<n \leqslant x} g(n, \tilde{f}, \mathbf{b}, \alpha, \beta, c-1, \varepsilon) \psi(n+(c-1) \varepsilon+\mu, v(n+(c-1) \varepsilon+\mu)) \\
= & \sum_{u=0}^{N} \sum_{\substack{c<n \leqslant x \\
q^{u} \| n+(c-1) \varepsilon+\mu}} g(n, \widetilde{f}, \mathbf{b}, \alpha, \beta, c-1, \varepsilon) \psi(n+(c-1) \varepsilon+\mu, u) \\
= & \sum_{u=0}^{N} \sum_{\substack{c<n \leqslant x \\
n+(c-1) \varepsilon+\mu \equiv 0}} g\left(n, \tilde{f}, \mathbf{b o d} q^{u}, \alpha, \beta, c-1, \varepsilon\right) \psi(n+(c-1) \varepsilon+\mu, u) \\
& -\sum_{u=0}^{N} \sum_{\substack{c<n \leqslant x \\
n+(c-1) \varepsilon+\mu \equiv 0} \bmod q^{u+1}} g(n, \widetilde{f}, \mathbf{b}, \alpha, \beta, c-1, \varepsilon) \psi(n+(c-1) \varepsilon+\mu, u) .
\end{aligned}
$$


En utilisant la relation d'orthogonalité classique à savoir

$$
\frac{1}{m} \sum_{j=0}^{m-1} e\left(\frac{j(b-a)}{m}\right)= \begin{cases}1 & \text { si } a \equiv b \quad \bmod m \\ 0 & \text { sinon }\end{cases}
$$

on obtient

$$
\begin{aligned}
G(\beta, c)= & \sum_{u=0}^{N} \sum_{l=0}^{q^{u}-1} \sum_{c<n \leqslant x} \frac{1}{q^{u}} g\left(n, \tilde{f}, \mathbf{b}, \alpha, \beta+\frac{l}{q^{u}}, c-1, \varepsilon\right) \\
& \times \psi(n+(c-1) \varepsilon+\mu, u) e\left(\frac{((c-1) \varepsilon+\mu) l}{q^{u}}\right) \\
& -\sum_{u=0}^{N} \sum_{l=0}^{q^{u+1}-1} \sum_{c<n \leqslant x} \frac{1}{q^{u+1}} g\left(n, \tilde{f}, \mathbf{b}, \alpha, \beta+\frac{l}{q^{u+1}}, c-1, \varepsilon\right) \\
& \times \psi(n+(c-1) \varepsilon+\mu, u) e\left(\frac{((c-1) \varepsilon+\mu) l}{q^{u+1}}\right) .
\end{aligned}
$$

Il est à noter que pour $u$ et $\mathbf{b}$ fixé la suite $\psi(n+(c-1) \varepsilon+\mu, u)$ est $q^{u+1}$ périodique (la périodicité de $\psi$ vient du fait que $\gamma(\mathbf{b}, ., u)$ est $q^{u+1}$-périodique), ce qui nous conduit à écrire

$$
\begin{aligned}
G(\beta, c)= & \sum_{u=0}^{N} \sum_{l=0}^{q^{u}-1} \sum_{i=(1-c) \varepsilon}^{q^{u+1}+(1-c) \varepsilon-1} \sum_{\substack{c<n \leqslant x \\
c \equiv i \\
\text { mod } q^{u+1}}} \frac{1}{q^{u}} g\left(n, \tilde{f}, \mathbf{b}, \alpha, \beta+\frac{l}{q^{u}}, c-1, \varepsilon\right) \\
& \times \psi(i+(c-1) \varepsilon+\mu, u) e\left(\frac{((c-1) \varepsilon+\mu) l}{q^{u}}\right) \\
& -\sum_{u=0}^{N} \sum_{l=0}^{q^{u+1}-1} \sum_{i=(1-c) \varepsilon}^{q^{u+1}+(1-c) \varepsilon-1} \sum_{\substack{c \equiv i \leqslant x \\
c<n}} \frac{1}{q^{u+1}} \\
& \times g\left(n, \tilde{f}, \mathbf{b}, \alpha, \beta+\frac{l}{q^{u+1}}, c-1, \varepsilon\right) \\
& \times \psi(i+(c-1) \varepsilon+\mu, u) e\left(\frac{((c-1) \varepsilon+\mu) l}{q^{u+1}}\right) .
\end{aligned}
$$

En utilisant encore une autre fois la relation d'orthogonalité (2.22) et l'expression 
de $G$ dans (2.20), on obtient

$$
\begin{aligned}
G(\beta, c)= & \sum_{u=0}^{N} \sum_{l=0}^{q^{u}-1} \sum_{k=0}^{q^{u+1}-1} \sum_{i=(1-c) \varepsilon}^{q^{u+1}+(1-c) \varepsilon-1} \frac{1}{q^{2 u+1}} \psi(i+(c-1) \varepsilon+\mu, u) \\
& \times e\left(\frac{((c-1) \varepsilon+\mu) l}{q^{u}}-\frac{i k}{q^{u+1}}\right) G\left(\beta+\frac{q l+k}{q^{u+1}}, c-1\right) \\
& -\sum_{u=0}^{N} \sum_{l=0}^{q^{u+1}-1} \sum_{k=0}^{q^{u+1}-1} \sum_{i=(1-c) \varepsilon}^{q^{u+1}} \frac{1}{q^{2 u+2}} \psi(i+(c-1) \varepsilon+\mu, u) \\
& \times e\left(\frac{((c-1) \varepsilon+\mu) l}{q^{u+1}}-\frac{i k}{q^{u+1}}\right) G\left(\beta+\frac{l+k}{q^{u+1}}, c-1\right) .
\end{aligned}
$$

Dans la dernière somme de chaque quadruple somme, on effectue un changement de compteur $i$ par $i+(c-1) \varepsilon$, on obtient

$$
\begin{aligned}
G(\beta, c)= & \sum_{u=0}^{N} \sum_{l=0}^{q^{u}-1} \sum_{k=0}^{q^{u+1}-1} \sum_{i=0}^{q^{u+1}-1} \frac{1}{q^{2 u+1}} G\left(\beta+\frac{q l+k}{q^{u+1}}, c-1\right) \\
& \times \psi(i+\mu, u) e\left(\frac{((c-1) \varepsilon+\mu) l}{q^{u}}-\frac{(i-(c-1) \varepsilon) k}{q^{u+1}}\right) \\
& -\sum_{u=0}^{N} \sum_{l=0}^{q^{u+1}-1} \sum_{k=0}^{q^{u+1}-1} \sum_{i=0}^{q^{u+1}-1} \frac{1}{q^{2 u+2}} G\left(\beta+\frac{l+k}{q^{u+1}}, c-1\right) \\
& \times \psi(i+\mu, u) e\left(\frac{((c-1) \varepsilon+\mu) l}{q^{u+1}}-\frac{(i-(c-1) \varepsilon) k}{q^{u+1}}\right) .
\end{aligned}
$$

En utilisant l'expression de $\psi$ dans (2.21) et de $F_{\mathbf{b}, u, \varepsilon}$ dans (2.2), on obtient

$$
\begin{aligned}
G(\beta, c)= & \sum_{u=0}^{N} \sum_{l=0}^{q^{u}-1} \sum_{k=0}^{q^{u+1}-1} \frac{1}{q^{u}} e\left(\frac{((c-1) \varepsilon+\mu) l}{q^{u}}+\frac{((c-1) \varepsilon) k}{q^{u+1}}-\varepsilon \alpha u\right) \\
& \times G\left(\beta+\frac{q l+k}{q^{u+1}}, c-1\right) F_{\mathbf{b}, u, \varepsilon}(k) \\
& -\sum_{u=0}^{N} \sum_{l=0}^{q^{u+1}-1} \sum_{k=0}^{q^{u+1}-1} \frac{1}{q^{u+1}} e\left(\frac{((c-1) \varepsilon+\mu) l}{q^{u+1}}+\frac{((c-1) \varepsilon) k}{q^{u+1}}-\varepsilon \alpha u\right) \\
& \times G\left(\beta+\frac{l+k}{q^{u+1}}, c-1\right) F_{\mathbf{b}, u, \varepsilon}(k) .
\end{aligned}
$$


Ce qui donne

$$
\begin{aligned}
|G(\beta, c)| \leqslant & \sum_{u=0}^{N} \sum_{k=0}^{q^{u+1}-1} \mid G\left(\beta+\frac{q l+k}{q^{u+1}}, c-1|| F_{\mathbf{b}, u, \varepsilon}(k) \mid\right. \\
& +\sum_{u=0}^{N} \sum_{k=0}^{q^{u+1}-1} \mid G\left(\beta+\frac{l+k}{q^{u+1}}, c-1|| F_{\mathbf{b}, u, \varepsilon}(k) \mid,\right.
\end{aligned}
$$

or d'après l'hypothèse de récurrence et le lemme 2.3 , on obtient

$$
\begin{aligned}
|G(\beta, c)| & =|G(x, \tilde{f}, \mathbf{b}, \alpha, \beta, c, \varepsilon)| \\
& \ll\left(\log ^{2(c-1)+4} x\right) x^{1-\lambda_{q}(f)} \sum_{u=0}^{N}(u+2) q \log q \\
& \ll\left(\log ^{2 c+4} x\right) x^{1-\lambda_{q}(f)} .
\end{aligned}
$$

Théorème 2.10. Soient $q \geqslant 2, f \in \mathcal{F}^{\sharp}, T \in \mathbb{N}^{*} 0 \leqslant \theta<1$ et $\mathbf{c}=\left(c_{n}\right)_{n \geqslant 0}$ une suite d'entiers $(T, \theta)$-presque périodique. Alors pour tout $\beta \in \mathbb{R}$ et $x \geqslant 2$, on a

$$
H_{q}(x, f, \beta, \mathbf{c})=\sum_{\|c\|<n \leqslant x} \Lambda(n) e\left(f\left(n+c_{n}\right)+\beta n\right) \ll T\left(\log ^{2\|\mathbf{c}\|+4} x\right) x^{1-\eta_{q, f, \theta}},
$$

où $\|\mathbf{c}\|=\max \left|c_{i}\right|$, la constante implicite ne dépend que de $\|\mathbf{c}\|$ et $q$, la constante $\eta_{q, f, \theta}=\min \left(1-\theta, \lambda_{q}(f)\right)$.

\section{Démonstration.}

- En premier temps, on suppose que la suite $\mathbf{c}$ est constante $\left(c_{n}=c \in \mathbb{Z}\right.$, pour tout $n \in \mathbb{N}$ ). Le cas $c=0$ est traité dans le théorème E. Soit alors $c \in \mathbb{Z}^{*}$, posons $\varepsilon=\frac{c}{|c|}$. Il est clair que si $f=\sum_{k=1}^{q-1} a_{k}|n|_{k} \in \mathcal{F}^{\sharp}$ et $n+c>0$, alors $\widetilde{f}(n+c)=\widetilde{f}(n+\varepsilon|c|)$ ce qui donne avec (2.14), (2.20), la remarque $2.8 i i)$ et le lemme 2.9

$$
\begin{aligned}
H_{q}(x, \tilde{f}, \beta, \mathbf{c}) & =H_{q}(x, \tilde{f}, \beta, \varepsilon|\mathbf{c}|) \\
& =G\left(x, \tilde{f}, \mathbf{a}, a_{q-1}^{\tilde{q}}, \beta,|c|, \varepsilon\right) \\
& \ll\left(\log ^{2|| \mathbf{c}||+4} x\right) x^{1-\lambda_{q}(f)},
\end{aligned}
$$

et le lemme 2.7, nous permet de conclure.

- Soit maintenant $\mathbf{c}=\left(c_{n}\right)_{n \geqslant 0}$ une suite $T$-périodique (en particulier $(T, 0)$ presque périodique). On note dans ce qui suit $\mathbf{c}_{\mathbf{i}}=\left(c_{i n}\right)_{n \geqslant 0}$ pour dire que c'est la suite constante qui vaut $c_{i}$ sur $\mathbb{N}\left(c_{i n}=c_{i}\right.$, pour tout $\left.n \in \mathbb{N}\right)$. On peut supposer sans perte de généralité que la suite $\mathbf{c}$ est purement périodique, ce 
qui nous permet d'écrire avec la relation d'orthogonalité (2.22)

$$
\begin{aligned}
H_{q}(x, \tilde{f}, \beta, \mathbf{c}) & =\sum_{0 \leqslant i<T} \sum_{\substack{\|\mathbf{c}\|<n \leqslant x \\
n \equiv i}} \Lambda(n) e\left(\tilde{f}\left(n+c_{i}\right)+\beta n\right) \\
& =\frac{1}{T} \sum_{0 \leqslant i, l<T} e\left(-\frac{i l}{T}\right) \sum_{\|\mathbf{c}\|<n \leqslant x} \Lambda(n) e\left(\tilde{f}\left(n+c_{i}\right)+\left(\beta+\frac{l}{T}\right) n\right) \\
& =\frac{1}{T} \sum_{0 \leqslant i, l<T} e\left(-\frac{i l}{T}\right) H_{q}\left(x, \tilde{f}, \beta+\frac{l}{T}, \mathbf{c}_{\mathbf{i}}\right)+O(1) \\
& \ll T \max _{0 \leqslant i, l<T}\left|H_{q}\left(x, \tilde{f}, \beta+\frac{l}{T}, \mathbf{c}_{\mathbf{i}}\right)\right| \\
& \ll T\left(\log ^{2\|\mathbf{c}\|+4} x\right) x^{1-\eta_{q, f, 0}} .
\end{aligned}
$$

La dernière inégalité découle bien de la majoration (2.24).

Ce qui donne d'après le lemme 2.7

$$
H_{q}(x, f, \beta, \mathbf{c}) \ll T\left(\log ^{2\|\mathbf{c}\|+4} x\right) x^{1-\eta_{q, f, 0}} .
$$

- Finalement, on suppose que $\mathbf{c}=\left(c_{n}\right)_{n \geqslant 0}$ une suite d'entiers $(T, \theta)$-presque périodique, alors il existe $\mathbf{e}=\left(e_{n}\right) T$-périodique vérifiant (1.1), on peut supposer sans perte de généralité que $\|\mathbf{c}\| \geqslant\|\mathbf{e}\|$, alors on peut écrire

$$
\begin{aligned}
H_{q}(x, f, \beta, \mathbf{c})= & \sum_{\|\mathbf{c}\|<n \leqslant x} \Lambda(n) e\left(f\left(n+e_{n}\right)+\beta n\right) \\
& +\sum_{\substack{\|\mathbf{c}\|<n \leqslant x \\
c_{n} \neq e_{n}}} \Lambda(n) e\left(f\left(n+c_{n}\right)+\beta n\right) \\
& -\sum_{\substack{\|\mathbf{c}\|<n \leqslant x \\
n_{n} \neq e_{n}}} \Lambda(n) e\left(f\left(n+e_{n}\right)+\beta n\right) .
\end{aligned}
$$

En majorant dans les deux dernières sommes l'exponentielle par 1, on obtient

$$
\begin{aligned}
& H_{q}(x, f, \beta, \mathbf{c}) \ll\left|\sum_{\|\mathbf{c}\|<n \leqslant x} \Lambda(n) e\left(f\left(n+e_{n}\right)+\beta n\right)\right|+2 \sum_{\substack{\|\mathbf{c}\|<n \leqslant x \\
e_{n} \neq c_{n}}} \Lambda(n) \\
& \ll H_{q}(x, f, \beta, \mathbf{e})+2 \sum_{\substack{n \leqslant x \\
e_{n} \neq c_{n}}} \Lambda(n)+O(\|\mathbf{c}\|) \\
& \ll T\left(\log ^{2|| \mathbf{c} \|+4} x\right) x^{\left(1-\lambda_{q}(f)\right)}+x^{\theta} \log (x) .
\end{aligned}
$$

Il est à signaler que la dernière inégalité vient du fait que $\Lambda(n) \leqslant \log x$ (pour $n \leqslant x)$, la relation (1.1) et l'inégualité $(2.25)$ qui traite le cas périodique.

Remarque 2.11. Il est à signaler que le théorème 2.10 est en général non valable dans le cas où $\mathbf{c}=\left(c_{n}\right)$ est une suite bornée quelconque. À titre d'illustration, on 
considère $q=4, f=\frac{1}{2} s_{4} \in \mathcal{F}^{\sharp}, \beta \in \mathbb{Z}$ et $\mathbf{c}=\left(c_{n}\right)$ la suite définie par:

$$
c_{n}=\left\{\begin{array}{lll}
0 & \text { si } s_{4}(n) \equiv 0 & \bmod 2, \\
1 & \text { si } s_{4}(n) \equiv 1 & \bmod 2 \text { et } n \equiv 0 \\
-1 & \text { sinon. }
\end{array}\right.
$$

Il est clair que pour tout $n \in \mathbb{N}$, on a $s_{4}\left(n+c_{n}\right) \equiv 0 \bmod 2$, ce qui donne $f\left(n+c_{n}\right) \in \mathbb{N}$, et par conséquent

$$
\sum_{1 \leqslant n \leqslant x} \Lambda(n) e\left(f\left(n+c_{n}\right)+\beta n\right)=\sum_{1 \leqslant n \leqslant x} \Lambda(n)=x+O\left(\frac{x}{\log x}\right) .
$$

Il est à noter qu'on pourra améliorer l'estimation donnée par le théorème 2.10 dans le cas où $f \in \mathcal{F}^{\sharp} \bigcap \mathcal{F}_{0}$.

Théorème 2.12. Soient $q \geqslant 2, f \in \mathcal{F}^{\sharp} \cap \mathcal{F}_{0}, T \in \mathbb{N}^{*} 0 \leqslant \theta<1$ et $\mathbf{c}=\left(c_{n}\right)_{n \geqslant 0}$ une suite d'entiers $(T, \theta)$-presque périodique. Alors pour tout $\beta \in \mathbb{R}$ et $x \geqslant 2$, on a

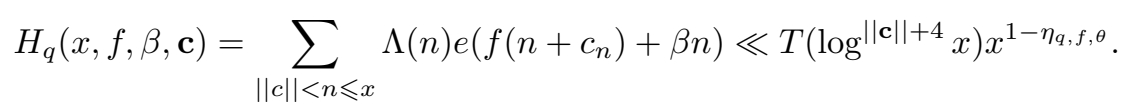

où $\|\mathbf{c}\|=\max \left|c_{i}\right|$ la constante implicite ne dépend que de $\|\mathbf{c}\|$ et $q$, la constante $\eta_{q, f, \theta}=\min \left(1-\theta, \lambda_{q}(f)\right)$.

Démonstration. Comme dans le théorème 2.10, il suffit de montrer l'estimation demandée dans ce théorème dans le cas des suites constantes positives ou nulles. Montrons ce théorème par récurrence sur $c$.

Soit $\mathbf{a}=\left(a_{k}\right)_{0 \leqslant k<q}$ une suite à valeurs dans $\mathbb{R}$. Si $f=\sum_{0 \leqslant k<q} a_{k}|\cdot|_{k} \in$ $\mathcal{F}^{\sharp} \cap \mathcal{F}_{0}$, alors la suite $\gamma(\mathbf{a}, n, i)$ donnée par (2.1) est une suite constante et vaut

$$
\gamma(\mathbf{a}, n, i)=a_{1}-a_{0}, \quad \text { pour tout } n, i \in \mathbb{N}
$$

ce qui donne pour $\varepsilon \in\{ \pm 1\}$ et l'expression de $\psi$ dans (2.21)

$$
\psi(w, u)=e\left(\varepsilon\left(a_{1}-a_{0}\right)-\varepsilon a_{q-1} u\right),
$$

ce qui confirme que $\psi(w, u)$ est constante par rapport à $w$. Ce qui donne avec (2.23) avec le choix de $\mathbf{b}=\mathbf{a}$ et $\alpha=\tilde{a_{q-1}}$.

$$
\begin{aligned}
|G(\beta, c)| & =\left|H_{q}(x, \tilde{f}, \beta, \mathbf{c})\right| \\
& \leqslant \sum_{u=0}^{T} \frac{1}{q^{u}} \sum_{l=0}^{q^{u}-1}\left|G\left(\beta+\frac{l}{q^{u}}, c-1\right)\right|+\sum_{u=0}^{T} \frac{1}{q^{u+1}} \sum_{l=0}^{q^{u+1}-1}\left|G\left(\beta+\frac{l}{q^{u+1}}, c-1\right)\right| \\
& \ll\left(\log ^{|| \mathbf{c} \|+4} x\right) x^{1-\lambda_{q}(f)},
\end{aligned}
$$

la dernière majoration est due à l'hypothèse de récurrence. Finalement, le lemme 2.7 nous permet de conclure. 
Dans la suite, on va étendre la notion de presque périodicité de la suite $\mathbf{c}=\left(c_{n}\right)$ dans le théorème 2.10 à une autre classe de suites régulières.

Définition 2.13. Une suite $\mathbf{c}=\left(c_{n}\right)$ à valeurs dans un ensemble fini $A$ est dite $q$-automatique s'il existe une suite $\mathbf{d}=\left(d_{n}\right)$ point fixe d'un morphisme $\sigma$ définie sur un alphabet fini $B$ de longueur constante qui vaut $q$ (i.e. pour tout $b \in B$, $\sigma(b)=b_{i_{1}} \ldots b_{i_{q}}$, avec $b_{i_{j}} \in B$ ) et une projection $\tau: B \rightarrow A$ telle que $\tau(\mathbf{d})=\mathbf{c}$.

Pour la notion d'automaticité, on peut consulter ([1]) ou ([8]).

Exemple 2.14. La suite de Thue-Morse $\mathbf{c}=\left(c_{n}\right)$, définie par $c_{n}=s_{2}(n)(\bmod 2)$ est une suite 2-automatique engendrée par le morphisme $\sigma$ définie sur $\{0,1\}$ avec $\sigma(0)=01$ et $\sigma(1)=10$, donc $\sigma^{3}(0)=011010, \sigma^{6}(0)=011010011001 \cdots$ et $\mathbf{c}=\sigma^{\infty}=\lim \sigma^{n}(0)$.

Il est à noter que la suite $\mathbf{c}=\left(c_{n}\right)$, définie par $c_{n}=s_{q}(n)(\bmod q)$ est aussi $q$-automatique engendrée par le morphisme $\sigma$ définie sur $\{0,1, \cdots, q-1\}$ avec $\sigma(0)=012 \cdots q-1, \sigma(1)=12 \cdots(q-1) 0$, et pour $i \in\{0,1, \cdots, q-1\} \sigma(i)=$ $i(i+1) \cdots(q-1) 01 \cdots(i-1)$.

D'une manière générale, on peut vérifier facilement que si $q$ est une base entière de numération alors la suite $\left(|n|_{k} \bmod q\right)_{n \geqslant 0}$ est $q$-automatique, pour tout $k \geqslant 1$, ce qui nous permet de conclure que toute fonction digitale $g(n)=$ $\sum_{1 \leqslant k<q} b_{k}|n|_{k} \in \mathcal{F}$ telle que $b_{k} \in \mathbb{Z}$ est $q$-automatique modulo $q$.

Notation 2.15. On note $\mathcal{F}^{b}$ l'ensemble des fonctions digitales $f(n)=$ $\sum_{1 \leqslant j<q} a_{j}|n|_{j}$, tels que les $a_{i}$ sont dans $\mathbb{Z}$.

Une question naturelle qui se pose à savoir: Est ce que le théorème 2.10 reste toujours vrai si la suite $\mathbf{c}=\left(c_{n}\right)$ appartient à une famille large de suite $q$-automatique. Un pas dans cette direction est la proposition suivante

Proposition 2.16. Soient $q \geqslant 2, g \in \mathcal{F}^{b}$ et $f \in \mathcal{F}$ telle que $f+\frac{1}{q} g \in \mathcal{F}^{\sharp}$ et $\mathbf{c}=\left(c_{n}\right)=(g(n) \bmod q)$. Alors pour tout $\beta \in \mathbb{R}$ et $x \geqslant 2$, on a

$$
H(x, f, \beta, \mathbf{c})=\sum_{\|\mathbf{c}\|<n \leqslant x} \Lambda(n) e\left(f\left(n+c_{n}\right)+\beta n\right) \ll\left(\log ^{2\|\mathbf{c}\|+4} x\right) x^{1-\lambda_{q}(f)},
$$

la constante implicite ne dépend que de $\|\mathbf{c}\|$ et $q$, la constante $\lambda_{q}(f)$ est définie dans (1.2).

Démonstration. Soit $\mathbf{b}=\left(b_{k}\right)_{0 \leqslant k<q}$ et $f=\sum_{0 \leqslant j<q} b_{j}|\cdot|_{j}$.

$$
H_{q}(x, \tilde{f}, \beta, \mathbf{c})=\sum_{\|\mathbf{c}\|<n \leqslant x} \Lambda(n) e\left(\widetilde{f}\left(n+c_{n}\right)+\beta n\right) .
$$

En découpant la dernière somme suivant les entiers $n$ tels que $c_{n}=c$, pour $0 \leqslant$ 
$c<q$, on obtient

$$
\begin{aligned}
H_{q}(x, \tilde{f}, \beta, \mathbf{c}) & =\sum_{0 \leqslant c<q} \sum_{\substack{\|\mathbf{c}\|<n \leqslant x \\
c n=c}} \Lambda(n) e(\tilde{f}(n+c)+\beta n) \\
& =\sum_{0 \leqslant c<q} \sum_{\substack{\|\mathbf{c}\|<n \leqslant x \\
g(n) \equiv c \bmod q}} \Lambda(n) e(\tilde{f}(n+c)+\beta n) .
\end{aligned}
$$

Ce qui donne d'après la relation d'orthogonalité (2.22)

$$
H_{q}(x, \tilde{f}, \beta, \mathbf{c})=\frac{1}{q} \sum_{0 \leqslant c, l<q} \sum_{\|\mathbf{c}\|<n \leqslant x} \Lambda(n) e\left(\tilde{f}(n+c)+\beta n+\frac{g(n)-c}{q} l\right) .
$$

Ce qui donne d'après les expressions de $\tilde{f}(n+c)$ dans $(2.14)$, $\Gamma$ et $V$ dans $(2.15)$ et (2.16) ainsi que l'expression de $G$ dans (2.20)

$$
\begin{aligned}
H_{q}(x, \tilde{f}, \beta, \mathbf{c})= & \frac{1}{q} \sum_{0 \leqslant c, l<q} \sum_{\|\mathbf{c}\|<n \leqslant x} \Lambda(n) \\
& \times e\left(\left(\widetilde{f}+\frac{g}{q}\right)(n)+\beta n+\Gamma(\mathbf{b}, n, c, 1)-\tilde{b_{q-1}} V(n, c, 1)-\frac{c l}{q}\right) \\
= & \frac{1}{q} \sum_{0 \leqslant c, l<q} e\left(-\frac{c l}{q}\right) G\left(x \widetilde{f+\frac{g}{q}}, \mathbf{b}, \tilde{b_{q-1}}, \beta, c, 1\right) .
\end{aligned}
$$

La dernière égalité vient du fait que $\widetilde{f+\frac{g}{q}}=\widetilde{f}+\frac{g}{q}$ dès que $g \in \mathcal{F}^{b}$. Finalement, ce sont les lemmes 2.7 et 2.9 qui nous permettent de conclure le résultat attendu.

\subsection{Equirépartition modulo 1 de la suite $\left(\alpha f\left(p+c_{p}\right)\right)_{p \in \mathcal{P}}$}

Une suite réelle $\left(x_{n}\right)_{n \in \mathbb{N}}$ est dite équirépartie modulo 1 si pour tout intervalle $[a, b] \subset[0,1]$

$$
\lim _{N \rightarrow+\infty} \frac{1}{N} \sharp\left\{n \leqslant N ; x_{n} \in[a, b]\right\}=b-a
$$

D'après le critère de Weyl, la suite $\left(x_{n}\right)_{n \in \mathbb{N}}$ est équirépartie modulo 1 si et seulement si pour tout $h \in \mathbb{Z}^{*}$

$$
\sum_{n \leqslant N} e\left(h x_{n}\right)=o(N)
$$

Pour étudier l'équirépartition modulo 1 de la suite $\left(\alpha f\left(p+c_{p}\right)\right)_{p \in \mathcal{P}}$, nous emploierons le lemme suivant qui est un corollaire presque immédiat du théorème 2.10. 
Lemme 2.17. Soient $q \geqslant 2,0 \leqslant \theta<1$ et $f \in \mathcal{F}^{\sharp}$. Alors il existe $\sigma_{q, f, \theta}>0$ tel que pour toute suite $(T, \theta)$-presque périodique $\mathbf{c}=\left(c_{n}\right)$ à valeurs dans $\mathbb{Z}$, pour tout $\beta \in \mathbb{R}$ et $x \geqslant 2$, on a

$$
\Psi(x)=\sum_{\substack{\|\mathbf{c}\|<p \leqslant x \\ p \equiv k \\ \bmod m}} e\left(f\left(p+c_{p}\right)+\beta p\right) \ll T\left(\log ^{2|| \mathbf{c}||+3} x\right) x^{1-\sigma_{q, f, \theta}} .
$$

La constante implicite ne dépend que de $\|\mathbf{c}\|$ et $q$.

Démonstration. En utilisant la relation d'orthogonalité dans (2.22), on obtient

$$
\Psi(x)=\frac{1}{m} \sum_{\substack{|| \mathbf{c} \|<p<x \\ 0 \leqslant z<m}} e\left(f\left(p+c_{p}\right)+\left(\beta+\frac{z}{m}\right) p-\frac{k}{m} z\right) .
$$

Une intégration par partie standard (voir [18, Lemme 11, p. 1630]), on obtient

$$
\Psi(x) \ll \frac{1}{\log x} \max _{\|\mathbf{c}\|<t \leqslant x}\left|\sum_{\|\mathbf{c}\|<n<t} \Lambda(n) e\left(f\left(n+c_{n}\right)+\left(\beta+\frac{z}{m}\right) n\right)\right|+\sqrt{x},
$$

ce qui donne d'après le théorème 2.10 le résultat attendu pour le choix de $\sigma_{q, f, \theta}=$ $\min \left(\frac{1}{2}, \lambda_{q}(f), 1-\theta\right)$.

Lemme 2.18. Soient $q \geqslant 2, g \in \mathcal{F}^{b}$ et $f \in \mathcal{F}$ telle que $f+\frac{1}{q} g \in \mathcal{F}^{\sharp}$ et $\mathbf{c}=\left(c_{n}\right)=$ $(g(n) \bmod q)$. Alors pour tout $\beta \in \mathbb{R}$ et $x \geqslant 2$, on a

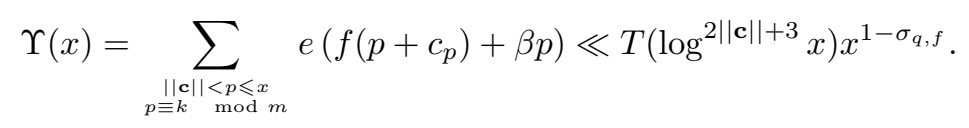

La constante implicite ne dépend que de $\|\mathbf{c}\|$ et $q$, la constante $\sigma_{q, f}=\min \left(\frac{1}{2}, \lambda_{q}(f)\right)$.

Démonstration. La même preuve que celle du lemme 2.17 quitte à utiliser la proposition 2.16 à la place du théorème 2.10 utilisé dans le lemme 2.17.

Soient $k$ et $m$ deux entiers premiers entre eux avec $m \geqslant 2$, on désigne par $\mathcal{P}_{k, m}$ l'ensemble des nombres premiers $p \equiv k(m)$.

Théorème 2.19. Soient $0 \leqslant \theta<1, k$ et $m$ deux entiers premiers entre eux, $m \geqslant 2$, $\mathbf{c}=\left(c_{n}\right)$ une suite $(T, \theta)$-presque périodique à valeurs dans $\mathbb{Z}$ et $f=$ $\sum_{0 \leqslant k<q} a_{j}|\cdot|_{j} \in \mathcal{F}^{b}$.

1) Si la suite $a_{0}, \cdots, a_{q-1}$ est constante, alors la suite $\left(\alpha f\left(p+c_{p}\right)\right)_{p \in \mathcal{P}_{k, m}}$ n'est pas équirépartie modulo 1.

2) Si la suite $a_{0}, \cdots, a_{q-1} n^{\prime}$ est pas constante, alors la suite $\left(\alpha f\left(p+c_{p}\right)\right)_{p \in \mathcal{P}_{k, m}}$ est équirépartie modulo 1 si et seulement si $\alpha \in \mathbb{R} \backslash \mathbb{Q}$. 
Démonstration. 1) Supposons que la suite $\left(\alpha f\left(p+c_{p}\right)\right)_{p \in \mathcal{P}_{k, m}}$ est équirépartie modulo 1 , alors d'après le critère de Weyl, on a

$$
\frac{1}{\pi(x, k, m)} \sum_{\substack{\|\mathbf{c}\|<p<x \\ p \equiv k}} e\left(\alpha f\left(p+c_{p}\right)\right)=o(1)
$$

et donc pour $j$ suffisamment grand,

$$
\sum_{\substack{q^{j-1}+|| \mathbf{c}|| \leqslant p<q^{j}-|| \mathbf{c}|| \\ p \equiv k \\ \bmod m}} e\left(\alpha f\left(p+c_{p}\right)\right)=o\left(\pi\left(q^{j}, k, m\right)\right) .
$$

Comme $a_{0}=\cdots=a_{q-1}$ et que $j=\left\lfloor\frac{\log \left(p+c_{p}\right)}{\log q}\right\rfloor$ quand $q^{j-1}+\|\mathbf{c}\| \leqslant p<q^{j}-\|\mathbf{c}\|$, alors

$$
\begin{aligned}
& \sum_{\substack{q^{j-1}+\left|\mathbf{c}\left\|\leqslant p<q^{j}-|| \mathbf{c}\right\| \\
p \equiv k\right.}} e\left(\alpha f\left(p+c_{p}\right)\right)=\sum_{\substack{\bmod m \\
q^{j-1}+\left|\mathbf{c}\left\|\leqslant p<q^{j}-\mid \mathbf{c}\right\| \\
p \equiv k(m)\right.}} e\left(\alpha a_{0}\left\lfloor\frac{\log \left(p+c_{p}\right)}{\log q}\right\rfloor\right) \\
& =e\left(\alpha a_{0} j\right)\left(\pi\left(q^{j}-\|c\|, k, m\right)\right. \\
& \left.-\pi\left(q^{j-1}+\|c\|, k, m\right)\right)+O(1) \\
& =e\left(\alpha a_{0} j\right)\left(\pi\left(q^{j}, k, m\right)-\pi\left(q^{j-1}, k, m\right)\right)+O(1) \text {. }
\end{aligned}
$$

Comme $e\left(\alpha a_{0} j\right) \neq 0$, on obtient

$$
\frac{\pi\left(q^{j}, k, m\right)-\pi\left(q^{j-1}, k, m\right)}{\pi\left(q^{j}, k, m\right)}=o(1)
$$

une contradiction avec le théorème des nombres premiers en progression arithmétiques qui fournit

$$
\lim _{j \rightarrow \infty} \frac{\pi\left(q^{j}, k, m\right)-\pi\left(q^{j-1}, k, m\right)}{\pi\left(q^{j}, k, m\right)}=1-\frac{1}{q} .
$$

2) Si $\alpha$ est rationnel, alors la suite $\left(\alpha f\left(p+c_{p}\right)\right)_{p \in \mathcal{P}_{k, m}}$ ne comporte qu'un nombre fini de terme modulo 1 et n'est donc équirépartie modulo 1 . Etant donné $\alpha \in \mathbb{R} \backslash \mathbb{Q}$ et $h \in \mathbb{Z}^{*}$, il est clair que $h \alpha f \in \mathcal{F}^{\sharp}$, ce qui donne avec le lemme 2.17

$$
\sum_{\substack{|| \mathbf{c} \|<p \leqslant x \\ p \equiv k \bmod m}} e\left(h \alpha f\left(p+c_{p}\right)\right)=o(\pi(x, k, m)) \quad\left(h \in \mathbb{Z}^{*}\right) .
$$

C'est le critère de Weyl qui nous permet de conclure.

On pourra établir un résultat analogue à celui du théorème 2.19 , dans le cas où $\mathbf{c}=\left(c_{n}\right)$ est une suite définie dans la proposition 2.16 tout en utilisant le lemme 2.18 . 
Théorème 2.20. Soient $q \geqslant 2, g \in \mathcal{F}^{b}, f=\sum_{0 \leqslant k<q} a_{k}|\cdot|_{k} \in \mathcal{F}^{b}$ et $\mathbf{c}=\left(c_{n}\right)=$ $(g(n) \bmod q)$.

1) Si la suite $a_{0}, \cdots, a_{q-1}$ est constante, alors la suite $\left(\alpha f\left(p+c_{p}\right)\right)_{p \in \mathcal{P}}$ n'est pas équirépartie modulo 1.

2) Si la suite $a_{0}, \cdots, a_{q-1}$ n'est constante, alors la suite $\left(\alpha f\left(p+c_{p}\right)\right)_{p \in \mathcal{P}}$ est équirépartie modulo 1 si et seulement si $\alpha \in \mathbb{R} \backslash \mathbb{Q}$.

\subsection{Application au problème ternaire de Goldbach}

En 1937 Vinogradov [22] a donné une réponse complète au problème original de Goldbach pour tout entier impair suffisamment grand sa méthode repose sur l'estimation des sommes d'exponentielles de type $\sum_{p \leqslant N} e(\alpha p)$. Il montre que pour tout $A>0$ fixé

$$
r(N)=\sum_{\substack{n_{1}, n_{2}, n_{3} \\ n_{1}+n_{2}+n_{3}=N}} \Lambda\left(n_{1}\right) \Lambda\left(n_{2}\right) \Lambda\left(n_{3}\right)=\mathcal{G}(N) N^{2}+O_{A}\left(\frac{N^{2}}{\log ^{A} N}\right)
$$

où

$$
\mathcal{G}(N)=\frac{1}{2} \prod_{p \mid N}\left(1-(p-1)^{-2}\right) \prod_{p \nmid N}\left(1+(p-1)^{-3}\right) .
$$

Il est à signaler que si $N$ est un entier impair suffisamment grand on a $\mathcal{G}(N) \gg 1$.

Usant de la notation $\mathbf{x}$ pour désigner le triplet $\left(x_{1}, x_{2}, x_{3}\right)$. Nous introduisons pour $\mathbf{c}=\left(\mathbf{c}_{1}, \mathbf{c}_{2}, \mathbf{c}_{3}\right)$ un triplet de suites $\left(T_{i}, \theta_{i}\right)$-presque périodiques avec $\mathbf{c}_{\mathbf{i}}=$ $\left(c_{i n}\right)$ pour $i \in\{1,2,3\}$.

$$
r(N, \mathbf{q}, \mathbf{a}, \mathbf{b}, \mathbf{c})=\sum_{\substack{n_{1}, n_{2}, n_{3} \\ n_{1}+n_{2}+n_{3}=N, n_{i}+c_{i n}>0 \\ f_{i}\left(n_{i}+c_{i n_{i}}\right) \equiv a_{i}\left(b_{i}\right), i \in\{1,2,3\}}} \Lambda\left(n_{1}\right) \Lambda\left(n_{2}\right) \Lambda\left(n_{3}\right) .
$$

Pour bien étudier le comportement de $r(N, \mathbf{q}, \mathbf{a}, \mathbf{b}, \mathbf{c})$, nous introduisons la notion d'entier caractéristique puis nous faisons appel à un lemme. Soit alors $q \geqslant 2$ une base de numération entière, nous introduisons la classe $\mathcal{F}_{q}^{+}$des fonctions $f$ de la forme

$$
f=\sum_{1 \leqslant k<q} a_{k}|\cdot|_{k}, \text { avec } a_{1}, \cdots, a_{q-1} \in \mathbb{Z} \quad \text { et }\left(a_{1}, \cdots, a_{q-1}\right)=1 .
$$

Dans [16], les auteurs ont introduit la notion d'entier caractéristique à savoir

Définition 2.21. Soient $q, b \geqslant 2$ et $f \in \mathcal{F}_{q}^{+}$, on appelle entier caractéristique de $f, b$ et $q$ et on note $d=d_{f, b, q}$ le plus grand diviseur positif de $(b, q-1)$ tel que pour tout $n \in \mathbb{N}$,

$$
f(n) \equiv f(1) s_{q}(n) \equiv f(1) n \bmod d .
$$




\section{Remarque 2.22.}

i) La relation (2.26) est équivalente à

$$
a_{k} \equiv a_{1} k \quad \bmod d \quad(1 \leqslant k<q) .
$$

ii) Il est à signaler que si $(b, q-1)=1$ alors $d=1$, la réciproque est fausse.

iii) Comme $f \in \mathcal{F}_{q}^{+}$, il est clair d'après i) que $(f(1), d)=1$.

iv) Il est à noter d'après iii) que si $d=d_{f, b, q} \geqslant 2$, alors $f(1)$ est inversible modulo $d$.

Lemme 2.23. Soient $0 \leqslant \theta<1, q, b \geqslant 2, f \in \mathcal{F}_{q}^{+}$, et $d=d_{f, b, q}$ définie dans (2.26). Alors il existe $\sigma_{f, b, q}>0$ tel que pour toute suite $\mathbf{c}=\left(c_{n}\right)(T, \theta)$-presque périodique à valeurs dans $\mathbb{Z}$ et pour tout $\beta \in \mathbb{R}, j \in J=\left\{0 \leqslant j<b, \frac{b}{d} \nmid j\right\}$ et $x \geqslant 2$,

$$
\sum_{\|\mathbf{c}\|<p \leqslant x} e\left(\frac{j}{b} f\left(p+c_{p}\right)+\beta p\right) \ll T\left(\log ^{2\|c\|+3} x\right) x^{1-\sigma_{q, f, \theta, b}} .
$$

La constante implicite ne dépend que de $\|\mathbf{c}\|$ et $q$, la constante $\sigma_{q, f, \theta, b}$ est donnée par

$$
\sigma_{q, f, \theta, b}=\min \left(\min _{\frac{b}{(b, q-1)} \nmid j} \sigma_{q, \frac{j}{b} f, \theta}, \min _{1 \leqslant r<\frac{(b, q-1)}{d}} \sigma_{q, \frac{r}{(b, q-1)} f, \theta}\right),
$$

( $\sigma_{q, f, \theta}$ est définie dans le lemme 2.17).

Démonstration. On utilise la męme preuve faite dans [16, Proposition 5], quitte à utiliser à chaque fois le lemme 2.17 qui remplace la [16, Proposition 4 ].

Théorème 2.24. Pour $i \in\{1,2,3\}$, soient $q_{i}, b_{i}$ deux entiers $\geqslant 2$, ainsi que $f_{i} \in$ $\mathcal{F}_{q_{i}}^{+}$et $\mathbf{c}_{i}=\left(c_{i n}\right)$ une suite $\left(T_{i}, \theta_{i}\right)$-presque périodique. Si l'entier caractéristique $d_{f_{i}, b_{i}, q_{i}}=1$ (défini en (2.26)), alors il existe $\tau_{\mathbf{q}, \mathbf{f}, \theta, \mathbf{b}}>0$ tel que pour $n \geqslant 2$

$$
r(N, \mathbf{q}, \mathbf{a}, \mathbf{b}, \mathbf{c})=\frac{r(N)}{b_{1} b_{2} b_{3}}+O\left(\max T_{i}\left(\log ^{2 \max \left\|\mathbf{c}_{i}\right\|+5} N\right) N^{2-\tau_{\mathbf{q}, \mathbf{f}, \theta, \mathbf{b}}}\right),
$$

où la constante implicite ne dépend que de $\left\|\mathbf{c}_{\mathbf{i}}\right\|$ et $\mathbf{q}$, avec $\tau_{\mathbf{q}, \mathbf{f}, \theta, \mathbf{b}}=$ $\min _{i=1,2,3} \sigma_{q_{i}, f_{i}, \theta_{i}, b_{i}}\left(\sigma_{q_{i}, f_{i}, \theta_{i}, b_{i}}\right.$ est définie dans le lemme 2.23).

En particulier, il existe un entier $N_{0}$ dépendant de $\mathbf{f}, \mathbf{q}, \mathbf{b}$ et $\mathbf{c}$ tel que tout entier impair $N>N_{0}$ s'écrit sous la forme

$$
N=p_{1}+p_{2}+p_{3} \quad \text { avec } f_{i}\left(p_{i}+c_{i p_{i}}\right) \equiv a_{i}\left(b_{i}\right) \quad \text { pour } i \in\{1,2,3\} .
$$

où les $p_{i}$ sont des nombres premiers $>\left\|\mathbf{c}_{i}\right\|$.

Démonstration. Il est à noter que Martin-Mauduit-Rivat [16] ont montré le théorème 2.24 pour le cas où les $\mathbf{c}_{\mathbf{i}}$ sont nulles. Nous optons ici pour la même variante utilisée dans leur démonstration, quitte à utiliser à chaque fois le lemme 2.23 à la place de la [16, Proposition 5]. 


\section{Théorème de type Gelfond}

L'objectif de cette partie est l'étude de quelques propriétés statistiques de l'ensemble $U_{a, b, \mathbf{c}, f}=\left\{p+c_{p}: p>\|\mathbf{c}\|, f\left(p+c_{p}\right) \equiv a \bmod b\right\}$ où $f \in \mathcal{F}_{q}^{+}$et $\mathbf{c}=\left(c_{n}\right)$ est une suite $(T, \theta)$-presque périodique. Soit alors,

$$
\begin{aligned}
U_{a, b, \mathbf{c}, f}(x) & =\left\{n \leqslant x: n \in U_{a, b, \mathbf{c}, f}\right\} \\
U_{a, b, \mathbf{c}, f}(k, m, x) & =\left\{p+c_{p} \in U_{a, b, \mathbf{c}, f}(x): p \equiv k \quad \bmod m\right\} .
\end{aligned}
$$

Pour étudier les ensembles $U_{a, b, \mathbf{c}, f}(x)$ et $U_{a, b, \mathbf{c}, f}(k, m, x)$, nous emploierons le lemme suivant

Lemme 3.1 (Lemme chinois généralisé). Soient $\alpha_{1}, \cdots, \alpha_{t} \in \mathbb{Z}, n_{1}, \cdots, n_{t} \in$ $\mathbb{Z}^{*}$. Le système d'équations

$$
\begin{cases}x \equiv \alpha_{1} & \bmod n_{1}, \\ \vdots & \\ x \equiv \alpha_{t} & \bmod n_{t},\end{cases}
$$

admet une solution si, seulement si, $\alpha_{i} \equiv \alpha_{j} \bmod \left(n_{i}, n_{j}\right)$, pour tous $i, j=$ $1, \cdots, t$. Dans ce cas, la solution est unique modulo $\operatorname{ppcm}\left(n_{i}, i=1, \cdots, t\right)$.

Théorème 3.2. Soient $b, q \geqslant 2, f \in \mathcal{F}_{q}^{+}, d=d_{f, q, b}$ l'entier caractéristique défini dans (2.26) et $\mathbf{c}=\left(c_{n}\right)$ une suite $(T, \theta)$-presque périodique, $\mathbf{e}=\left(e_{l}\right)$ la suite associée à $\mathbf{c}$ vérifiant la relation (1.1). On pose ı l'inverse arithmétique de $f(1)$ modulo $d$ si $d \geqslant 2$. Alors, pour tous $a \in \mathbb{Z}$ et $x \geqslant 2$

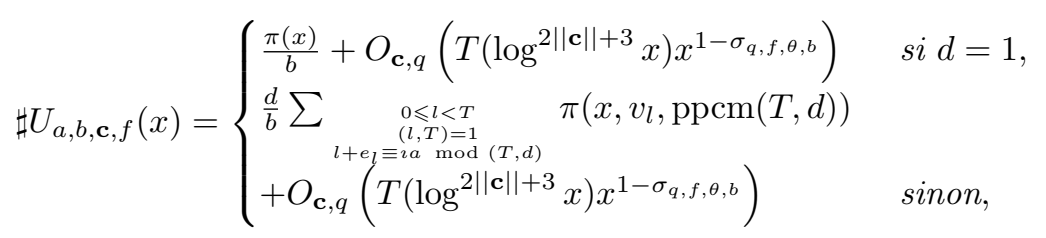

où $\sigma_{q, f, \theta, b}$ est défini dans le lemme 2.23 et $v_{l}$ est une solution du système de congruences

$$
\left\{\begin{array}{l}
v_{l} \equiv l \quad \bmod T \\
v_{l} \equiv a \imath-e_{l} \quad \bmod d
\end{array}\right.
$$

Démonstration. Soit $J=\left\{0 \leqslant j<b, \frac{b}{d} \nmid j\right\}$.

$$
\sharp U_{a, b, \mathbf{c}, f}(x)=\frac{1}{b} \sum_{0 \leqslant j<b} \sum_{\substack{p+c_{p} \leqslant x \\ p>\|\mathbf{c}\|}} e\left(\frac{f\left(p+c_{p}\right)-a}{b} j\right)=S_{1}+S_{2},
$$

avec

$$
S_{1}=\frac{1}{b} \sum_{j \in J} \sum_{\substack{p+c_{p} \leqslant x \\ p>\|\mathbf{c}\|}} e\left(\frac{f\left(p+c_{p}\right)-a}{b} j\right)
$$


et

$$
S_{2}=\frac{1}{b} \sum_{j \notin J} \sum_{\substack{p+c_{p} \leqslant x \\ p>\|\mid \mathbf{c}\|}} e\left(\frac{f\left(p+c_{p}\right)-a}{b} j\right) .
$$

D'une part, d'après le lemme 2.23

$$
S_{1} \ll T\left(\log ^{2\|\mathbf{c}\|+3} x\right) x^{1-\sigma_{q, f, \theta, b}} .
$$

D'autre part, si $j \notin J$, alors $j=\frac{u b}{d}, 0 \leqslant u<d$, donc

$$
\begin{aligned}
S_{2}= & \frac{1}{b} \sum_{\substack{p+c_{p} \leqslant x \\
p>\|\mathbf{c}\|}} \sum_{0 \leqslant u<d} e\left(\frac{u}{d}\left(f\left(p+c_{p}\right)-a\right)\right) \\
= & \frac{1}{b} \sum_{\substack{p+e_{p} \leqslant x \\
p>\|\mathbf{c}\|}} \sum_{0 \leqslant u<d} e\left(\frac{u}{d}\left(f\left(p+e_{p}\right)-a\right)\right) \\
& -\frac{1}{b} \sum_{\substack{p+e_{p} \leqslant x \\
p>\|\mid \mathbf{c}\|, c_{p} \neq e_{p}}} \sum_{0 \leqslant u<d} e\left(\frac{u}{d}\left(f\left(p+e_{p}\right)-a\right)\right) \\
& +\frac{1}{b} \sum_{\substack{p+c_{p} \leqslant x \\
p>\|\mid \mathbf{c}\|, c_{p} \neq e_{p}}} \sum_{0 \leqslant u<d} e\left(\frac{u}{d}\left(f\left(p+c_{p}\right)-a\right)\right) .
\end{aligned}
$$

En majorant l'exponentielle par 1 dans les deux dernières doubles sommes et en utilisant la relation (1.1), on obtient

$$
S_{2}=\frac{1}{b} \sum_{\substack{p+e_{p} \leqslant x \\ p>\|\mathbf{c}\|}} \sum_{0 \leqslant u<d} e\left(\frac{u}{d}\left(f\left(p+e_{p}\right)-a\right)\right)+O\left(x^{\theta}\right),
$$

Ce qui donne avec la relation (2.26)

$$
S_{2}=\frac{1}{b} \sum_{\substack{p+e_{p} \leqslant x \\ p>\|\mathbf{c}\|}} \sum_{\substack{0 \leqslant u<d \\ d}} e\left(\frac{u}{d}\left(f(1)\left(p+e_{p}\right)-a\right)\right)+O\left(x^{\theta}\right) .
$$

- Si $d=1$, alors d'après la relation (3.2)

$$
S_{2}=\frac{\pi(x)}{b}+O\left(x^{\theta}\right)
$$

- Supposons maintenant que $d \geqslant 2$, comme e est purement périodique, alors

$$
e_{\alpha T+l}=e_{l}, \quad \text { pour tous } \alpha, l \in \mathbb{N},
$$


ce qui donne avec la relation (3.2) et la relation d'orthogonalité (2.22),

$$
\begin{aligned}
& S_{2}=\frac{1}{b} \sum_{\substack{0 \leqslant l<T \\
(l, T)=1}} \sum_{\substack{p+e_{l} \leqslant x \\
p \equiv l \text { mod } T \\
p>|| \mathbf{c}||}} \sum_{0 \leqslant u<d} e\left(\frac{u}{d}\left(f(1)\left(p+e_{l}\right)-a\right)\right)+O\left(x^{\theta}\right) \\
& =\frac{d}{b} \sum_{\substack{0 \leqslant l<T \\
(l, T)=1}} \sum_{\substack{p+e_{l} \leqslant x \\
p \equiv l \\
\bmod T \\
f(1)\left(p+e_{l}\right) \equiv a}} 1+O\left(x^{\theta}\right)
\end{aligned}
$$

comme $d \geqslant 2$, alors d'après la remarque 2.22, f(1) est inversible modulo $d$, alors

$$
S_{2}=\frac{d}{b} \sum_{\substack{0 \leqslant l<T \\(l, T)=1}} \sum_{\substack{p \leqslant x \\ p \equiv l \\ p \equiv a \nu-e_{l} \bmod T \\ \bmod d}} 1+O\left(x^{\theta}\right) .
$$

Ce qui donne avec le lemme 3.1 et la définition de $v_{l}$ dans (3.1), le résultat attendu.

Proposition 3.3. Soient $b, q \geqslant 2,0 \leqslant \theta<1, f \in \mathcal{F}_{q}^{+}, d=d_{f, q, b}$ l'entier caractéristique défini dans (2.26) tel que $d \geqslant 2$ et $\mathbf{c}=\left(c_{n}\right)$ une suite $(T, \theta)$-presque périodique, $\mathbf{e}=\left(e_{l}\right)$ la suite associée à $\mathbf{c}$ vérifiant la relation (1.1). On pose $\imath_{d}=\imath$ l'inverse arithmétique de $f(1)$ modulo $d$. Si pour tout $l$ : $0 \leqslant l<T,(l, T)=1$, $l+e_{l} \neq a \imath \bmod (d, T)$, alors

$$
\sharp U_{a, b, \mathbf{c}, f}(x)=O\left(x^{\theta}\right) .
$$

\section{Démonstration.}

$$
\begin{aligned}
U_{a, b, \mathbf{c}, f}(x) & \subset U_{a, d, \mathbf{c}, f}(x)=\left\{p+c_{p} \leqslant x: p>\|\mathbf{c}\|, f\left(p+c_{p}\right) \equiv a \bmod d\right\} \\
& =\left\{p+c_{p} \leqslant x: p>\|\mathbf{c}\|, f(1)\left(p+c_{p}\right) \equiv a \bmod d\right\} \\
& =\left\{p+c_{p} \leqslant x: p>\|\mathbf{c}\|, p+c_{p} \equiv a \imath \bmod d\right\}
\end{aligned}
$$

or d'après les relations (1.1) et (2.26), on a

$$
\begin{aligned}
\sharp U_{a, d, \mathbf{c}, f}(x) & =\sharp\left\{p+e_{p} \leqslant x: p>\|\mathbf{c}\|, p+e_{p} \equiv a \imath \bmod d\right\}+O\left(x^{\theta}\right) \\
& =\sharp\left\{p+e_{p} \leqslant x: p>\|\mathbf{e}\|, p+e_{p} \equiv a \imath \bmod d\right\}+O\left(x^{\theta}\right) \\
& =\sharp U_{a, d, \mathbf{e}, f}(x)+O\left(x^{\theta}\right) .
\end{aligned}
$$

Comme

$$
\begin{aligned}
& U_{a, d, \mathbf{e}, f}(x) \subset \bigcup_{0 \leqslant l<T}\left\{p+e_{l} \leqslant x: p>\|\mathbf{e}\|,\right. \\
&\left.\operatorname{avec} p \equiv a \imath-e_{l} \quad \bmod d \text { et } p \equiv l \bmod T\right\} .
\end{aligned}
$$


Il est clair que si $l+e_{l} \neq a \imath \bmod (d, T)$, alors

$$
\sharp\left\{p+e_{l} \leqslant x: p \equiv a \imath-e_{l} \bmod d, p \equiv l \bmod T\right\}=0,
$$

ce qui donne avec les relations (3.5) et (3.6)

$$
\sharp U_{a, d, \mathbf{e}, f}(x) \leqslant \pi(T) .
$$

En employant l'écriture de $U_{a, d, \mathbf{c}, f}(x)$ dans (3.4) et les relations (3.3) et (3.7), il vient

$$
\sharp U_{a, b, \mathbf{c}, f}(x)=O\left(x^{\theta}\right) .
$$

Dans la suite nous étudions l'équirépartition de la suite $(\alpha p)_{p+c_{p} \in U_{a, b, \mathbf{c}, f}}$ dès qu'il existe $l$ : $0 \leqslant l<T$, tel que $(l, T)=1$ et $l+e_{l} \equiv a \imath \bmod (d, T)$.

Théorème 3.4. Soient $b, q \geqslant 2,0 \leqslant \theta<1, a \in \mathbb{Z}, f \in \mathcal{F}_{q}^{+}, d=d_{f, q, b}$ l'entier caractéristique défini dans (2.26) et $\mathbf{c}=\left(c_{n}\right)$ une suite $(T, \theta)$-presque périodique et $\mathbf{e}=\left(e_{l}\right)$ la suite associée à $\mathbf{c}$ vérifiant la relation (1.1). On pose $\imath_{d}=\imath$ l'inverse arithmétique de $f(1)$ modulo $d$ si $d \geqslant 2$ et 0 si $d=1$. On suppose qu'il existe $0 \leqslant l<T$, tel que

$$
(l, T)=1 \text { et } l+e_{l} \equiv a \imath \bmod (d, T) .
$$

Alors la suite $(\alpha p)_{p+c_{p} \in U_{a, b, \mathbf{c}, f}}$ est équirépartie modulo 1 si et seulement si $\alpha \in$ $\mathbb{R} \backslash \mathbb{Q}$.

Démonstration. Soient $h \in \mathbb{Z}^{*}, \alpha \in \mathbb{R} \backslash \mathbb{Q}$ et $J=\left\{0 \leqslant j<b, \frac{b}{d} \nmid j\right\}$.

$$
\begin{aligned}
\sum_{p+c_{p} \in U_{a, b, \mathbf{c}, f}} e(h \alpha p)= & \sum_{\substack{\|\mathbf{c}\|<p \leqslant x \\
f\left(p+c_{p}\right) \equiv a}} e(h \alpha p)+O(1) \\
= & \frac{1}{b} \sum_{j=0}^{b-1} \sum_{\| \mathbf{m o d} b} e\left(\frac{j}{b}\left(f\left(p+c_{p}\right)-a\right)+h \alpha p\right)+O(1) \\
\leqslant & \frac{1}{b} \sum_{j \in J}\left|\sum_{\|\mathbf{c}\|<p \leqslant x} e\left(\frac{j}{b} f\left(p+c_{p}\right)+h \alpha p\right)\right| \\
& +\frac{1}{b} \sum_{j \notin J}\left|\sum_{\|\mathbf{c}\|<p \leqslant x} e\left(\frac{j}{b} f\left(p+c_{p}\right)+h \alpha p\right)\right|+O(1) .
\end{aligned}
$$

D'après le lemme 2.23

$$
\frac{1}{b} \sum_{j \in J}\left|\sum_{\|\mathbf{c}\|<p \leqslant x} e\left(\frac{j}{b} f\left(p+c_{p}\right)+h \alpha p\right)\right| \ll\left(\log ^{2|| \mathbf{c}||+3}\right) x^{1-\sigma_{q, f, \theta, b}},
$$


Maintenant, pour $j \notin J$, alors $j=\frac{u b}{d}$ avec $0 \leqslant u<d$ et d'après la relation (2.26), il vient

$$
\begin{aligned}
\sum_{\|\mathbf{c}\|<p \leqslant x} e\left(\frac{j}{b} f\left(p+c_{p}\right)+h \alpha p\right)= & \sum_{\|\mathbf{c}\|<p \leqslant x} e\left(\frac{u}{d} f\left(p+c_{p}\right)+h \alpha p\right) \\
= & \sum_{\substack{\|\mathbf{c}\|<p \leqslant x\\
}} e\left(\frac{u}{d} f(1)\left(p+e_{p}\right)+h \alpha p\right) \\
& -\sum_{\substack{\|\mathbf{c}\|<p \leqslant x \\
c p \neq e_{p}}} e\left(\frac{u}{d} f(1)\left(p+e_{p}\right)+h \alpha p\right) \\
& +\sum_{\substack{\|\mathbf{c}\|<p \leqslant x \\
c_{p} \neq e_{p}}} e\left(\frac{u}{d} f(1)\left(p+c_{p}\right)+h \alpha p\right) .
\end{aligned}
$$

En majorant l'exponentielle par 1 dans les deux dernières sommes et en utilisant la relation $(1.1)$, on obtient

$$
\begin{aligned}
\Sigma_{j} & =\sum_{\|\mathbf{c}\|<p \leqslant x} e\left(\frac{j}{b} f\left(p+c_{p}\right)+h \alpha p\right) \\
& =\sum_{\|\mathbf{c}\|<p \leqslant x} e\left(\frac{u}{d} f(1)\left(p+e_{p}\right)+h \alpha p\right)+O\left(x^{\theta}\right) .
\end{aligned}
$$

En décomposant la dernière somme dans (3.11) suivant les congruences à $p$ modulo $T$, puis en utilisant la relation d'orthogonalité (2.22), on obtient

$$
\begin{aligned}
& \Sigma_{j}=\sum_{\|\mathbf{c}\|<p \leqslant x} e\left(\frac{j}{b} f\left(p+c_{p}\right)+h \alpha p\right) \\
& =\sum_{\substack{0 \leqslant l<T \\
(l, T)=1}} e\left(\frac{u e_{l} f(1)}{d}\right) \sum_{\substack{\|\mathbf{c}\|<p \leqslant x \\
p \equiv l=0 \\
\bmod T}} e\left(\left(\frac{u}{d} f(1)+h \alpha\right) p\right)+O\left(x^{\theta}\right) \\
& =\frac{1}{T} \sum_{\substack{0 \leqslant l<T \\
(l, T)=1 \\
0 \leqslant k<T}} e\left(\frac{u e_{l} f(1)}{d}-\frac{l k}{T}\right) \sum_{\|\mathbf{c}\|<p \leqslant x} e\left(\left(\frac{u}{d} f(1)+h \alpha+\frac{k}{T}\right) p\right)+O\left(x^{\theta}\right) \\
& \ll \frac{1}{T} \sum_{\substack{0 \leqslant l<T \\
(l, T)=1 \\
0 \leqslant k<T}}\left|\sum_{\|\mathbf{c}\|<p \leqslant x} e\left(\left(\frac{u}{d} f(1)+h \alpha+\frac{k}{T}\right) p\right)\right|+O\left(x^{\theta}\right) .
\end{aligned}
$$

Comme $\alpha \in \mathbb{R} \backslash \mathbb{Q}$, alors $\frac{u}{d} f(1)+h \alpha+\frac{k}{T}$ l'est aussi, or d'après le théorème de Vinogradov qui assure l'équirépartition de $(\alpha p)_{p \in \mathcal{P}}$ dès que $\alpha \in \mathbb{R} \backslash \mathbb{Q}$ et le critère de Weyl, on obtient

$$
\sum_{\|\mathbf{c}\|<p \leqslant x} e\left(\left(\frac{u}{d} f(1)+h \alpha+\frac{k}{T}\right) p\right)=o(\pi(x)),
$$


ce qui donne

$$
\begin{aligned}
& \frac{1}{b} \sum_{j \notin J}\left|\sum_{\|\mathbf{c}\|<p \leqslant x} e\left(\frac{j}{b} f\left(p+c_{p}\right)+h \alpha p\right)\right| \leqslant \frac{1}{b} \sum_{j \notin J}\left|\Sigma_{j}\right| \\
& \leqslant \frac{1}{b} \sum_{0 \leqslant u<d}\left|\sum_{\|\mathbf{c}\|<p \leqslant x} e\left(\left(\frac{u}{d} f(1)+h \alpha+\frac{k}{T}\right) p\right)\right|=o(\pi(x)) .
\end{aligned}
$$

Finalement, la condition (3.8) entraîne, en vertu du théorème 3.2

$$
\sharp U_{a, b, \mathbf{c}, f}(x) \approx \pi(x),
$$

ce qui donne avec $(3.9),(3.10)$ et $(3.13)$

$$
\sum_{p+c_{p} \in U_{a, b, \mathbf{c}, f}} e(h \alpha p)=o\left(U_{a, b, \mathbf{c}, f}(x)\right),
$$

c'est le critère de Weyl qui nous permet de conclure.

Théorème 3.5. Soient $b, m, q \geqslant 2, f \in \mathcal{F}_{q}^{+}, d=d_{f, q, b}$ l'entier caractéristique défini dans (2.26) et $\mathbf{c}=\left(c_{n}\right)$ une suite $(T, \theta)$-presque périodique et $\mathbf{e}=\left(e_{l}\right)$ la suite associée à c vérifiant la relation (1.1). On pose $\imath$ l'inverse arithmétique de $f(1)$ modulo $d$ si $d \geqslant 2$. Alors, pour tous $a, k \in \mathbb{Z}$ et $x \geqslant 2$

$\sharp U_{a, b, \mathbf{c}, f}(k, m, x)=$

$$
\begin{cases}\frac{\pi(x, k, m)}{b}+O_{\mathbf{c}, q}\left(T\left(\log ^{2\|\mathbf{c}\|+3} x\right) x^{1-\sigma_{q, f, \theta, b}}\right) & \text { si } d=1, \\ \sum_{\substack{0 \leqslant l<T,(l, T)=1 \\ k \equiv a n-e_{l} \bmod (m, d) \\ l+e_{l} \equiv a_{2} \bmod (T, d) \\ l \equiv k \bmod (m, T)}} \pi\left(x, v_{l}, \operatorname{ppcm}(T, d, m)\right) & \\ +O_{\mathbf{c}, q}\left(T\left(\log ^{2\|\mathbf{c}\|+3} x\right) x^{1-\sigma_{q, f, \theta, b}}\right) & \text { sinon, }\end{cases}
$$

où $\sigma_{q, f, \theta, b}$ est défini dans le lemme 2.23 et $v_{l}$ est une solution du système de congruences

$$
\left\{\begin{array}{l}
v_{l} \equiv l \quad \bmod T \\
v_{l} \equiv a \imath-e_{l} \quad \bmod d \\
v_{l} \equiv k \quad \bmod m
\end{array}\right.
$$

Démonstration. Soit $J=\left\{0 \leqslant j<b, \frac{b}{d} \nmid j\right\}$.

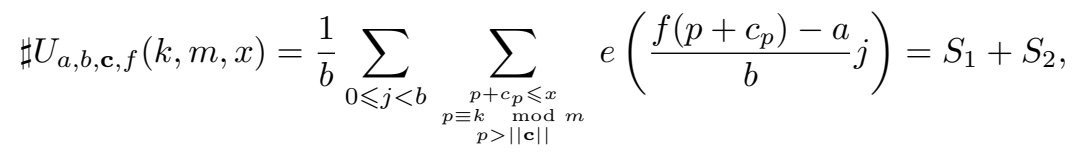

avec

$$
S_{1}=\frac{1}{b} \sum_{j \in J} \sum_{\substack{p+c_{p} \leqslant x \\ p \equiv k \\ p>|| \mathbf{c} \|}} e\left(\frac{f\left(p+c_{p}\right)-a}{b} j\right)
$$


et

$$
S_{2}=\frac{1}{b} \sum_{j \notin J} \sum_{\substack{p+c_{p} \leqslant x \\ p \equiv k \text { mod } \\ p>\|\mathbf{c}\|}} e\left(\frac{f\left(p+c_{p}\right)-a}{b} j\right)
$$

D'une part, d'après le lemme 2.23

$$
S_{1} \ll T\left(\log ^{2|| \mathbf{c} \|+3} x\right) x^{1-\sigma_{q, f, \theta, b}} .
$$

D'autre part, si $j \notin J$, alors $j=\frac{u b}{d}, 0 \leqslant u<d$, donc

$$
S_{2}=\frac{1}{b} \sum_{\substack{p+c_{p} \leqslant x \\ p \equiv k \\ p>\| \mathbf{c} \mid}} \sum_{0 \leqslant u<d} e\left(\frac{u}{d}\left(f\left(p+c_{p}\right)-a\right)\right) .
$$

Ce qui donne avec la relation (2.26)

$$
\begin{aligned}
& S_{2}=\frac{1}{b} \sum_{\substack{p+c_{p} \leqslant x \\
p \equiv k}} \sum_{0 \leqslant u<d} e\left(\frac{u}{d}\left(f(1)\left(p+c_{p}\right)-a\right)\right)+O(1) \\
& =\frac{1}{b} \sum_{\substack{p+e_{p} \leqslant x \\
p \equiv k}} \sum_{0 \leqslant u<d} e\left(\frac{u}{d}\left(f(1)\left(p+e_{p}\right)-a\right)\right) \\
& -\frac{1}{b} \sum_{\substack{p+e_{p} \leqslant x \\
\text { mod } m \\
c_{p} \neq e_{p}}} \sum_{0 \leqslant u<d} e\left(\frac{u}{d}\left(f(1)\left(p+e_{p}\right)-a\right)\right) \\
& +\frac{1}{b} \sum_{\substack{p+c_{p} \leqslant x \\
p \equiv k \text { mod } m \\
c_{p} \neq e_{p}}} \sum_{0 \leqslant u<d} e\left(\frac{u}{d}\left(f(1)\left(p+c_{p}\right)-a\right)\right)+O(1) .
\end{aligned}
$$

En majorant l'exponentielle par 1 dans les deux dernières doubles sommes et en utilisant la relation (1.1), on obtient

$$
S_{2}=\frac{1}{b} \sum_{\substack{p+e_{p} \leqslant x \\ p \equiv k \bmod m}} \sum_{0 \leqslant u<d} e\left(\frac{u}{d}\left(f(1)\left(p+e_{p}\right)-a\right)\right)+O\left(x^{\theta}\right) .
$$

- Si $d=1$, alors d'après la relation (3.16)

$$
S_{2}=\frac{\pi(x, k, m)}{b}+O\left(x^{\theta}\right)
$$

- Supposons maintenant que $d \geqslant 2$, comme e est purement périodique, alors

$$
e_{\alpha T+l}=e_{l}, \quad \text { pour tous } \alpha, l \in \mathbb{N} \text {. }
$$


En décomposant la somme dans (3.16) suivant les congruences à $p$ modulo $T$, puis en utilisant la relation d'orthogonalité (2.22), on obtient

$$
\begin{aligned}
& S_{2}=\frac{1}{b} \sum_{\substack{0 \leqslant l<T \\
(l, T)=1}} \sum_{\substack{p+e_{l} \leqslant x \\
p \equiv l \\
p \equiv k}} \sum_{\substack{\bmod T \\
\bmod m}} e\left(\frac{u}{d}\left(f(1)\left(p+e_{l}\right)-a\right)\right)+O\left(x^{\theta}\right)
\end{aligned}
$$

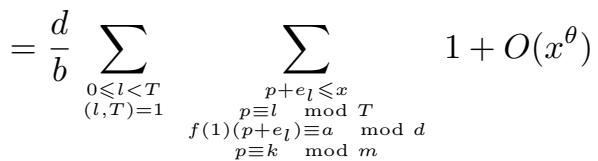

$$
\begin{aligned}
& =\frac{d}{b} \sum_{\substack{0 \leq l<T \\
(l, T)=1}} \sum_{\substack{p \leqslant x \\
p \equiv l=l \\
p \equiv a-e_{l} \bmod T \\
p \equiv k \bmod d}} 1+O\left(x^{\theta}\right) .
\end{aligned}
$$

Ce qui donne avec le lemme 3.1 et la définition de $v_{l}$ dans (3.15), le résultat attendu.

\section{Références}

[1] J.-P. Allouche, J. Shallit, Automatic sequences: Theory, applications, generalisations, Cambridge University Press, Cambridge, 2003.

[2] R. Bellman, H.N. Shapiro, On a problem in additive number theory, Ann. of Math. 49(2) (1948), 333-340.

[3] C. Dartyge, G. Tenenbaum, Sommes des chiffres de multiples d'entiers, Ann. Inst. Fourier 55(7) (2005), 2423-2474.

[4] H. Davenport, Multiplicative number theory, Graduate texts in Mathematics vol. 74, Springer-Verlag, New York, éd. third, 2000, revised and with a preface by Hugh L. Montgomery.

[5] M. Drmota, C. Mauduit, J. Rivat, Primes with an average sum of digits, Compos. Math. 145 (2009), no. 2, 271-292.

[6] M. Drmota, C. Mauduit, Weyl sums over integers with affine digit restrictions , J. Number Theory 130 (2010), no.11, 2404-2427.

[7] C.J. De La Vallée Poussin, Recherches analytiques sur la théorie des nombres pemiers, Brux. S. sc. 21 (1896), 183-256, 281-362, 363-397.

[8] S. Eilenberg, Automata, languages, and machines, Vol. Academic Press, (A subsidiary of Harcourt Brace Jovanovich, Publishers), Pure and Applied Mathematics, New York, 1974.

[9] C.G. Esseen, Fourier analysis of distribution functions, a mathematical study of Laplace-Gaussian law, Acta Math. 77 (1945), 1-125.

[10] N. Fine, The distribution of the sum of digits (modp), Bull. Amer. Math. Soc. 71 (1965), 651-652.

[11] E. Fouvry, C. Mauduit, Méthodes de crible et fonctions sommes des chiffres, Acta Arith. 77(4) (1996), 339-351.

[12] E. Fouvry, C. Mauduit, Sommes des chiffres et nombres presque premiers, Mathematische Annalen 305 (1996), 571-599. 
[13] A.O. Gel'fond, Sur les nombres qui ont des propriétés additives et multiplicatives données, Acta Arith. 13 (1968), 259-265.

[14] J. Hadamard, Sur la distribution des zéros de la fonction $\zeta(s)$ et ses conséquences arithmétiques, Bull. Soc. Math. France 24 (1896), 199-220.

[15] K. Mahler, The spectrum of an array and its application to the study of the translation properties of a simple class of arithmetical functions. II: On the translation properties of a simple class of arithmetical functions, J. Math. Phys. Mass. Inst. Techn. 6 (1927), 158-163.

[16] B. Martin, C. Mauduit, J. Rivat, Sur les chiffres des nombres premiers, http://iml.univ-mrs.fr/ rivat/publications.html

[17] C. Mauduit, Multiplicative properties of the Thue-Morse sequence, Period Math. Hungar. 43 (2001), 137-153.

[18] C. Mauduit, J. Rivat, Sur un probleme de Gelfond: la somme des chiffres des nombres premiers, Ann. of Math. 171 (2010), 1591-1646.

[19] C. Mauduit, J. Rivat, La somme des chiffres des carrés, Acta Mathematica 203 (2009), 107-148.

[20] C. Mauduit, A. Sárközy, On the arithmetic structure of sets characterized by sum of digits properties, J. Number Theory 61 (1996), no. 1, 25-38.

[21] C. Mauduit, A. Sárközy, On the arithmetic structure of the integers whose sum of digits is fixed, Acta Arith. 81 (1997), no. 2, 145-173.

[22] I.M. Vinogradov, The method of trigonometrical sums in the theory of numbers, translated from the Russian, revised and annotated by K. F. Roth and A. Davenport, Interscience, London, 1954.

Addresses: Mohamed Mkaouar and Najib Ouled Azaiez: Faculté des Sciences de Sfax, BP 1171, Sfax 3000, Tunisie;

Jörg M. Thuswaldner: Institut für Mathematik und Informationstechnologie, Abteilung für Mathematik und Statistik, Montanuniversität Leoben, Franz Josef Strasse 18, A-8700 Leoben, Austria.

E-mail: mohamed.mkaouar@fss.rnu.tn, najib.ouledazaiez@fss.rnu.tn, joerg.thuswaldner@unileoben.ac.at

Received: 20 April 2013; revised: 7 June 2013 